\title{
SN 2008in-BRIDGING THE GAP BETWEEN NORMAL AND FAINT SUPERNOVAE OF TYPE IIP
}

\author{
Rupak Roy ${ }^{1}$, Brijesh Kumar ${ }^{1}$, Stefano Benetti ${ }^{2}$, Andrea Pastorello ${ }^{3}$, Fang Yuan $^{4,5}$, Peter J. Brown ${ }^{6}$, \\ Stefan Immler $^{7,8}$, Timur A. FatKhullin ${ }^{9}$, Alexander S. Moskvitin ${ }^{9}$, Justyn Maund $^{10}$, Carl W. Akerlof $^{4}$, \\ J. Craig Wheeler ${ }^{11}$, Vladimir V. Sokolov ${ }^{9}$, Rorbert M. Quimby ${ }^{12}$, Filomena Bufano ${ }^{2}$, Brajesh Kumar $^{1,13}$, \\ Kuntal Misra $^{14,15}$, S. B. Pandey ${ }^{1,4}$, Nancy Elias-Rosa ${ }^{16}$, Peter W. A. Roming ${ }^{17}$, and Ram SagaR ${ }^{1}$

\begin{abstract}
We present optical photometric and low-resolution spectroscopic observations of the Type II plateau supernova (SN) 2008in, which occurred in the outskirts of the nearly face-on spiral galaxy M61. Photometric data in the X-ray, ultraviolet, and near-infrared bands have been used to characterize this event. The SN field was imaged with the ROTSE-IIIb optical telescope about seven days before the explosion. This allowed us to constrain the epoch of the shock breakout to JD $=2454825.6$. The duration of the plateau phase, as derived from the photometric monitoring, was $\sim 98$ days. The spectra of SN 2008in show a striking resemblance to those of the archetypal low-luminosity IIP SNe 1997D and 1999br. A comparison of ejecta kinematics of SN 2008in with the hydrodynamical simulations of Type IIP SNe by Dessart et al. indicates that it is a less energetic event $\left(\sim 5 \times 10^{50} \mathrm{erg}\right)$. However, the light curve indicates that the production of radioactive ${ }^{56} \mathrm{Ni}$ is significantly higher than that in the low-luminosity SNe. Adopting an interstellar absorption along the SN direction of $A_{V} \sim 0.3 \mathrm{mag}$ and a distance of $13.2 \mathrm{Mpc}$, we estimated a synthesized ${ }^{56} \mathrm{Ni}$ mass of $\sim 0.015 M_{\odot}$. Employing semi-analytical formulae derived by Litvinova and Nadezhin, we derived a pre-SN radius of $\sim 126 R_{\odot}$, an explosion energy of $\sim 5.4 \times 10^{50} \mathrm{erg}$, and a total ejected mass of $\sim 16.7 M_{\odot}$. The latter indicates that the zero-age main-sequence mass of the progenitor did not exceed $20 M_{\odot}$. Considering the above properties of SN 2008in and its occurrence in a region of sub-solar metallicity $([\mathrm{O} / \mathrm{H}] \sim$ $8.44 \mathrm{dex}$ ), it is unlikely that fall-back of the ejecta onto a newly formed black hole occurred in SN 2008in. We therefore favor a low-energy explosion scenario of a relatively compact, moderate-mass progenitor star that generates a neutron star.
\end{abstract}

Key words: supernovae: general - supernovae: individual (2008in)

Online-only material: color figures

\section{INTRODUCTION}

Core-collapse Type II supernovae (SNe) mark the violent death of stars with main-sequence masses greater than $8 M_{\odot}$, and as is indicated by the presence of hydrogen lines in their optical spectra, they originate from a progenitor star with a significant amount of hydrogen still intact (Eldridge \& Tout 2004). Of special interest are the Type II plateau SNe which are characterized by a "plateau" in their optical light curve and are more common, constituting about $75 \%$ of all Type II SNe (Smith et al. 2010). The IIP SNe show a wide range of plateau luminosities, plateau durations, expansion velocities, and nickel masses (Hamuy 2003), and these observational properties are connected with the explosion mechanism as well as the physical properties of the progenitor star such as ejected mass, explosion energy, and pre-SN radius (Nadyozhin 2003; Smartt et al. 2009). The IIP SNe are thought to result from progenitor masses in the range 8-25 $M_{\odot}$ (Heger et al. 2003) with an extended hydrogen envelope necessary to maintain the plateau phase. A detailed study of optical light curves and spectra of only a few nearby IIP SNe has been done so far and there exists a discrepancy in estimating the mass of their progenitors, e.g., for the three well-studied events (namely, 1999em, 2005cs, and 2004et), the determination of progenitor mass from the hydrodynamical modeling of their light curve is found to be higher than that estimated from pre-SN imaging (Utrobin et al. 2010; Bersten et al. 2011).

Recently, a number of "low-luminosity" Type IIP events have been discovered, viz., SNe 1999br, 1999eu, 1994N, 2001dc, 2005cs (Pastorello et al. 2004, 2009), 2008bk (Van Dyk et al. 
2010), and 2009md (Fraser et al. 2010). These events have explosion energy $\left(\sim 10^{50} \mathrm{erg}\right)$ and ejected ${ }^{56} \mathrm{Ni}$ mass $(2-7 \times$ $10^{-3} M_{\odot}$ ) both lower by a factor of 10 than normal, and low expansion velocity $\sim 1000 \mathrm{~km} \mathrm{~s}^{-1}$ (Pastorello et al. 2009 and references therein). The low-luminosity IIP SNe are debated because of the unknown nature of their progenitors. The first reported faint SN was SN 1997D (Turatto et al. 1998; Benetti et al. 2001), and the observed properties of its light curves and spectra were explained in terms of significant fall-back of ejected material on a newly formed black hole $(\mathrm{BH})$, created through the core-collapse of a massive progenitor ( $M \gtrsim 20 M_{\odot}$; Zampieri et al. 1998, 2003). Alternatively, SN 1997D was interpreted as the explosion of a less massive progenitor (8-12 $M_{\odot}$; Chugai \& Utrobin 2000), close in mass to the lower limit for stars that can undergo core-collapse. Heger et al. (2003) suggested that low-luminosity Type IIP events are electron capture $\mathrm{SNe}$ produced by low-mass progenitors giving rise to $\mathrm{ONeMg}$ cores. This is further supported through investigations of pre-explosion images (Maund \& Smartt 2005; Maund et al. 2005), though Eldridge et al. (2007) have ruled out the possibility of such a mechanism for the low-luminosity SN 2005 cs. According to the formalism of Heger et al. (2003) and Eldridge \& Tout (2004), no star having initial mass less than $22 M_{\odot}$ can form a $\mathrm{BH}$, which can quench the ejected material and produce a low-luminosity SN. Stars with masses above $25 M_{\odot}$, formed in metal-poor or slightly sub-solar metallicity regions, can produce low-luminosity, $\mathrm{BH}$-forming Type IIP $\mathrm{SNe}$. Type IIL/b events can be produced through this process from stars having masses $>25 M_{\odot}$ and generated in regions with solar (or super-solar) metallicity. So, the metallicity information at the $\mathrm{SN}$ location and the estimation of the initial mass are essential to constrain the triggering mechanisms of these explosions.

SN 2008in was discovered in the nearby galaxy M61 (NGC 4303). The first unfiltered CCD images of SN 2008in were taken by Koichi Itagaki on 2008 December 26.79 (all times in UT hereafter) and 27.69 at a magnitude of 14.9. Independent observations of this event by K. Kadota showed the transient at an unfiltered mag of 15.1. In addition, W. Wells recorded the SN on 2008 December 28.46 at $V$ - and $R$-band magnitudes of 14.3 and 13.2, respectively (Nakano et al. 2008). Low- and mid-resolution spectroscopic observations indicate an early discovery for SN 2008in (within 1-2 weeks after corecollapse). The spectra showed highly blueshifted $\mathrm{H} \alpha$ and $\mathrm{H} \beta$ absorptions (by $\sim 9000 \mathrm{~km} \mathrm{~s}^{-1}$ ) with weaker emission components (Chakraborti et al. 2008; Foley 2008; Stritzinger 2008). The presence of prominent P-Cygni profiles of Balmer lines leads to its classification as a Type II SN.

The broadband light curve and the initial spectral evolution of SN 2008in were similar to those of normal Type IIP SNe. However, from mid-plateau, the SN started to show a few spectral features (like $\mathrm{H} \alpha$ ) which are similar to underluminous events. SN 2008in was also observed in the radio with the Very Large Array (VLA) on 2008 December 31.40 UT in two frequency bands at 8.4601 and $22.4601 \mathrm{GHz}$ (Stockdale et al. 2008). Observation for the second epoch was further reported by Stockdale et al. (2009) on 2009 January 27. Interacting Type II SNe, like Type IIn events and a few Type IIP events (e.g., SN 2004et), are supposed to be strong sources of radio emission (for review see Weiler et al. 2002). However, both VLA observations produced null results for this proximate event.

In this paper, we present optical and near-infrared photometric and optical spectroscopic observations of SN 2008in. The photometric data cover a time span of about 410 days since the discovery. The Swift/XRT (Burrows et al. 2005) and Swift/ UVOT (Roming et al. 2005) data covering a time span of 60 days are also presented. In Sections 3 and 4, we study the photometric and spectroscopic evolutions, respectively. In Sections 5 and 6, we cover the estimates of distance, reddening, intrinsic color, and bolometric fluxes. The main physical parameters of the explosion and the mass of the progenitor are derived in Section 7 while a comparison of its properties with other $\mathrm{SNe}$ is given in Section 8. The conclusion of the paper is given in the last section. The epoch of explosion JD $=2454825.6$ (Section 3) is considered throughout the paper and the times of pre-/postexplosion are denoted by $-/+$ signs, respectively.

\section{OBSERVATION AND DATA REDUCTION}

\subsection{Photometric Observation}

The prompt follow-up of the event was carried out by the ground-based ROTSE-IIIb telescope ${ }^{18}$ having sensitivity in the wavelength region from 0.35 to $1.0 \mu \mathrm{m}$ with a peak around $0.6 \mu \mathrm{m}$ (Quimby et al. 2007). The SN was first detected in the ROTSE-IIIb images on 2008 December 24.45 and it was monitored at 58 phases until +115 days. The initial detections of the $\mathrm{SN}$ evaded the automated pipeline identification due to poor image quality and low signal-to-noise ratio $(\mathrm{S} / \mathrm{N})$. The data reduction was performed afresh on all the available ROTSE data. In order to remove the contamination of the true $\mathrm{SN}$ flux from the underlying galaxy, a pre-SN galaxy template was constructed from images taken in early 2008 and each SN frame was reduced using the galaxy-template subtraction scheme developed by Alard (2000). The point-spread function (PSF) photometry was performed at the SN location in the galaxy-template-subtracted images. The unfiltered instrumental magnitudes were calibrated using the USNO B1.0 $R$-band magnitudes of about 15 isolated stars. The light curve thus produced was found to be $0.15 \mathrm{mag}$ off from the Cousins $R$-band light curve produced by multiband observation carried out at ARIES (described below) and the ROTSE magnitudes were scaled accordingly.

The SN 2008in was also monitored with the Ultraviolet Optical Telescope (UVOT) on board Swift from +5 days to +60 days. The UVOT filters $u v w 2, u v m 2, u v w 1, u, b$, and $v$ have their effective wavelengths at 2030, 2231, 2634, 3501, 4329, and $5402 \AA$, respectively (Poole et al. 2008). The UVOT data reduction was performed following the prescriptions of Brown (2009). A 5" aperture is used to measure the counts for the coincidence loss correction whereas a $3^{\prime \prime}$ aperture was used for the photometry. For the filters $u v w 2, u v m 2$, and $u v w 1$, the last epoch (obtained about 60 days after explosion when the UV flux was very weak) data were used to subtract the galaxy light, while in the optical the SN remains much brighter than the underlying light so contamination was negligible. An aperture correction (based on an average PSF in Swift CALDB) as well as zero points from Poole et al. (2008) was applied to put the magnitudes on the UVOT photometric system. The UVOT magnitudes are listed in Table 1.

From +5 days to +416 days, the follow-up of SN 2008in in optical broadband Johnson $U B V$ and Cousins $R I$ was performed

\footnotetext{
18 The Robotic Optical Transient Search Experiment (ROTSE-III) is a set of four $45 \mathrm{~cm}$ fully robotic optical telescopes, installed at Siding Spring Observatory, Australia (ROTSE-IIIa), McDonald Observatory, Texas (ROTSE-IIIb), H.E.S.S. site, Namibia (ROTSE-IIIc), and TUBITAK National Observatory, Turkey (ROTSE-IIId; Akerlof et al. 2003; Yuan 2010).
} 
Table 1

The Swift/UVOT Photometric Observations of SN 2008in

\begin{tabular}{|c|c|c|c|c|c|c|c|c|}
\hline $\begin{array}{l}\text { UT Date } \\
(\mathrm{yy} / \mathrm{mm} / \mathrm{dd})\end{array}$ & $\begin{array}{c}\text { JD } \\
2454000+\end{array}$ & $\begin{array}{c}\text { Phase }^{\mathrm{a}} \\
\text { (day) }\end{array}$ & $\begin{array}{l}u v w 2 \\
(\mathrm{mag})\end{array}$ & $\begin{array}{l}\text { uvm2 } \\
\text { (mag) }\end{array}$ & $\begin{array}{l}u v w 1 \\
(\mathrm{mag})\end{array}$ & $\begin{array}{c}u \\
(\mathrm{mag})\end{array}$ & $\begin{array}{c}b \\
(\mathrm{mag})\end{array}$ & $\begin{array}{c}v \\
(\mathrm{mag})\end{array}$ \\
\hline $2008 / 12 / 30.08$ & 830.58 & 5.00 & $14.97 \pm 0.05$ & $14.65 \pm 0.04$ & $14.45 \pm 0.05$ & $14.12 \pm 0.04$ & $15.24 \pm 0.04$ & $15.20 \pm 0.04$ \\
\hline $2008 / 12 / 30.60$ & 831.11 & 5.53 & $15.13 \pm 0.05$ & $14.85 \pm 0.04$ & $14.54 \pm 0.04$ & $14.16 \pm 0.04$ & $15.20 \pm 0.04$ & $15.13 \pm 0.05$ \\
\hline $2008 / 12 / 31.96$ & 832.46 & 6.88 & $15.53 \pm 0.05$ & $15.28 \pm 0.09$ & $14.84 \pm 0.05$ & $14.32 \pm 0.04$ & $15.25 \pm 0.04$ & $15.15 \pm 0.06$ \\
\hline $2009 / 01 / 01.83$ & 833.30 & 7.72 & $15.72 \pm 0.05$ & $15.47 \pm 0.05$ & $15.01 \pm 0.05$ & $14.34 \pm 0.04$ & $15.30 \pm 0.04$ & $15.17 \pm 0.04$ \\
\hline $2009 / 01 / 03.54$ & 835.05 & 9.47 & $16.23 \pm 0.05$ & $16.07 \pm 0.05$ & $15.51 \pm 0.05$ & $14.52 \pm 0.04$ & $15.35 \pm 0.04$ & $15.25 \pm 0.04$ \\
\hline $2009 / 01 / 07.79$ & 839.29 & 13.71 & $17.87 \pm 0.08$ & $17.88 \pm 0.11$ & $16.96 \pm 0.07$ & $15.15 \pm 0.04$ & $15.49 \pm 0.04$ & $15.25 \pm 0.05$ \\
\hline $2009 / 01 / 11.15$ & 842.64 & 17.06 & $18.81 \pm 0.11$ & $18.66 \pm 0.14$ & $17.80 \pm 0.09$ & $15.74 \pm 0.04$ & $15.62 \pm 0.04$ & $15.27 \pm 0.04$ \\
\hline $2009 / 01 / 19.79$ & 851.30 & 25.72 & $\ldots$ & $\ldots$ & $18.67 \pm 0.16$ & $16.67 \pm 0.05$ & $16.00 \pm 0.04$ & $15.36 \pm 0.05$ \\
\hline $2009 / 01 / 22.00$ & 884.53 & 58.95 & $\ldots$ & $\ldots$ & $\ldots$ & $17.92 \pm 0.07$ & $16.77 \pm 0.05$ & $15.69 \pm 0.04$ \\
\hline $2009 / 01 / 22.73$ & 885.23 & 59.65 & $\ldots$ & $\ldots$ & $\ldots$ & $18.02 \pm 0.09$ & $16.75 \pm 0.05$ & $15.68 \pm 0.05$ \\
\hline
\end{tabular}

Note. ${ }^{a}$ With reference to the explosion epoch JD 2454825.6.

with the $1 \mathrm{~m}$ Sampurnanand Telescope (ST) at ARIES, Nainital. ${ }^{19}$ An identification chart showing the field of the galaxy M61 along with the locations of SN 2008in as well as the local standards is presented in Figure 1. The photometry is performed using standard tasks of IRAF ${ }^{20}$ and $D A O P H O T^{21}$ as described in the paper by Roy et al. (2011). Bias subtraction and flat fielding were performed on the raw frames and the cosmic removal was done using the LACOSMIC routine (van Dokkum 2001). As the SN lies in the outskirts of the galaxy on a relatively faint and smooth background, the photometry at the initial phases (mostly during the plateau phase when the $\mathrm{SN}$ is bright) is estimated using the profile fitting method. During nebular phases, when the $\mathrm{SN}$ becomes faint, the true $\mathrm{SN}$ flux is estimated using the galaxy template subtraction method following the procedures of Roy et al. (2011). As a galaxy template, we used post-explosion (+600 days) images observed on 2011 January 4 under good seeing conditions. Figure 2 shows an example of this procedure applied to a late-time $V$-band image of SN 2008in. In the template image of 2011 January 4, we can see a clear flux enhancement located approximately $5^{\prime \prime}$ away (a linear distance of $\sim 343 \mathrm{pc}$ ) from the SN position. This knot is also present in a deep image from the Sloan Digital Sky Survey (SDSS) and it is identified as a $\mathrm{H}$ II region in the NED catalog. The progenitor of SN 2008in may have a possible association with this starforming region. The field of SN 2008in was calibrated using Landolt (2009) standard stars of the field SA98 observed on the same night as the SN. A sample of 10 bright and isolated non-variable stars in the field of SN 2008in was used as local standards to derive the zero points for the SN at each epoch. The location and magnitudes of these local standards are listed in Table 2. The entire time span of photometric observation is about 410 days, and in Table 3 we report the $U B V R I$ photometry of the event.

From +4 days to +116 days, the SN 2008in was also observed in $J H K$ near-infrared (NIR) bands with the $0.6 \mathrm{~m} \mathrm{REM} / \mathrm{REMIR}$ at La Silla (Zerbi et al. 2004). The object was clearly visible

\footnotetext{
19 A $2048 \times 2048$ CCD camera mounted at the f/13 Cassegrain focus of the telescope has a square pixel of $24 \mu \mathrm{m}$ on a side, and with a plate scale of 0.38 arcsec per pixel, it covers a square area of $13^{\prime}$ on a side in the sky. The gain and readout noise of the CCD camera are 10 electrons per analog-to-digital unit and 5.3 electrons, respectively. To improve the $\mathrm{S} / \mathrm{N}$ and optimize the sampling, the observations were carried out in a binned mode of $2 \times 2$ pixel.

20 IRAF stands for Image Reduction and Analysis Facility distributed by the National Optical Astronomy Observatory which is operated by the Association of Universities for Research in Astronomy, Inc., under cooperative agreement with the National Science Foundation.

21 DAOPHOT stands for Dominion Astrophysical Observatory Photometry (Stetson 1987).
}

in the $J$ and $H$ passbands, while it remained undetected in $K$. The instrumental magnitudes were standardized using the Two Micron All Sky Survey standards available in the field of SN 2008in. The calibrated $J H$ magnitudes are listed in Table 4.

\subsection{X-ray Observations}

The Swift XRT observations were obtained simultaneously with the UVOT observations. To search for X-ray emission from SN 2008in, we extracted X-ray counts from a circular region with a 10 pixel radius $\left(23^{\prime \prime} .7\right.$; corresponding to the XRT on-axis $90 \%$ encircled energy radius) centered on the optical position of the SN. The background was extracted locally from a source-free region of $40^{\prime \prime}$ radius to account for the detector, sky background, and the diffuse emission from the host galaxy.

No X-ray source is detected in the merged $27.1 \mathrm{ks}$ XRT data obtained in photon-counting mode. The $3 \sigma$ upper limit to the XRT net count rate is $7.2 \times 10^{-4}$ counts s $^{-1}$, corresponding to an unabsorbed $\left(0.2-10 \mathrm{keV}\right.$ band) X-ray flux of $f_{0.2-10}<$ $3.4 \times 10^{-14} \mathrm{erg} \mathrm{cm}^{-2} \mathrm{~s}^{-1}$ and a luminosity of $L_{0.2-10}<7.0 \times$ $10^{38} \mathrm{erg} \mathrm{s}^{-1}$ for an adopted thermal plasma spectrum with a temperature of $k T=10 \mathrm{keV}$ (see Fransson et al. 1996 and references therein), a Galactic foreground column density of $N_{\mathrm{H}}=1.67 \times 10^{20} \mathrm{~cm}^{-2}$ (Dickey \& Lockman 1990), and a distance of 13.19 Mpc (Section 5).

\subsection{Optical Spectroscopic Observation}

Long-slit low-resolution spectra $(\sim 6$ to $14 \AA$ ) in the optical range $(0.33-1.0 \mu \mathrm{m})$ were collected at 11 phases from +7 days to +143 days, including five phases from the $2 \mathrm{~m} \mathrm{IGO}$, three phases from the $9.2 \mathrm{~m} \mathrm{HET}$, two phases from the $6 \mathrm{~m} \mathrm{BTA}$, and one epoch from the $3.6 \mathrm{~m} \mathrm{NTT}$. $^{22}$ A journal of spectroscopic observations is given in Table 5. The spectroscopic data acquired from IGO, NTT, and HET were reduced under the IRAF environment. Bias and flat-fielding were performed on all the frames. Cosmic-ray rejection was done using the Laplacian kernel detection method (van Dokkum 2001). All the data obtained from the BTA were reduced using programs in the IDL software environment.

The instrumental FWHM resolution of $2 \mathrm{~m}$ IGO spectra as measured from the [OI] $\lambda 5577$ emission skyline was found to lie between 6 and $10 \AA\left(\sim 322-510 \mathrm{~km} \mathrm{~s}^{-1}\right)$. Flux calibration was done using standard spectrophotometric fluxes from Hamuy et al. (1994), assuming a mean extinction for the site. For HET,

\footnotetext{
22 The full names of the telescope are provided in Table 5.
} 


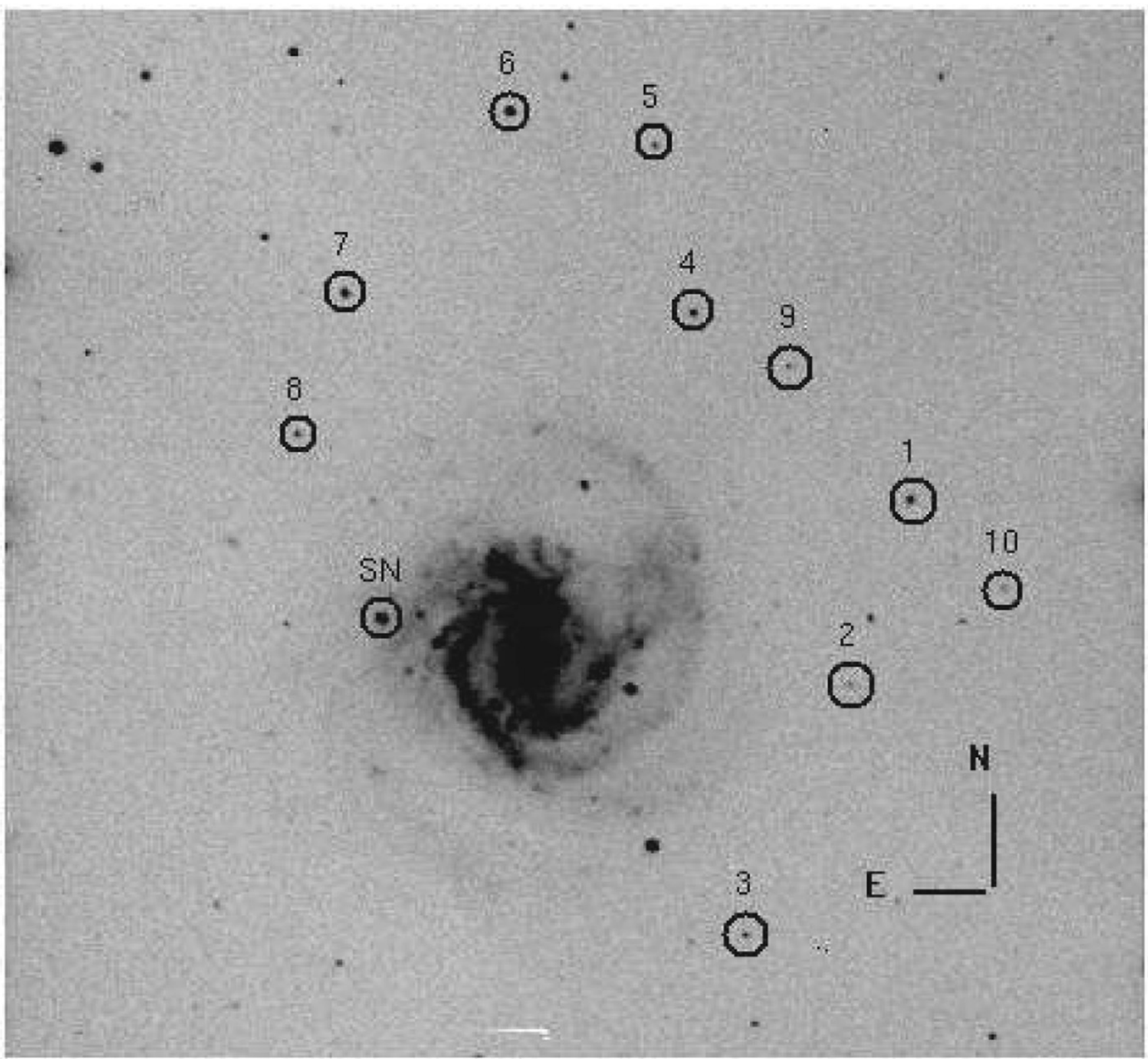

Figure 1. SN 2008in in M61. A $600 \mathrm{~s} V$-band image taken at phase +32 days from the $1 \mathrm{~m}$ ST ARIES, India and covering an area of about $10^{\prime} \times 10^{\prime}$ is shown. The location of SN 2008in and the local standard stars are marked with circles. North is up and east is to the left.

Table 2

The Photometric Magnitudes of Secondary Standard Stars in the Field of SN 2008in

\begin{tabular}{lccccccc}
\hline \hline $\begin{array}{l}\text { Star } \\
\text { ID }\end{array}$ & $\begin{array}{c}\alpha_{\mathrm{J} 2000} \\
(\mathrm{~h} \mathrm{~m} \mathrm{~s})\end{array}$ & $\begin{array}{c}\delta_{\mathrm{J} 2000} \\
\left({ }^{\prime} \quad{ }^{\prime \prime}\right)\end{array}$ & $\begin{array}{c}U \\
(\mathrm{mag})\end{array}$ & $\begin{array}{c}B \\
(\mathrm{mag})\end{array}$ & $\begin{array}{c}V \\
(\mathrm{mag})\end{array}$ & $\begin{array}{c}R \\
(\mathrm{mag})\end{array}$ & $\begin{array}{c}I \\
(\mathrm{mag})\end{array}$ \\
\hline 1 & 122138 & 043026 & $16.99 \pm 0.03$ & $17.69 \pm 0.03$ & $17.33 \pm 0.05$ & $16.96 \pm 0.06$ & $16.59 \pm 0.05$ \\
2 & 122140 & 042818 & $\ldots$ & $19.49 \pm 0.05$ & $18.38 \pm 0.04$ & $17.85 \pm 0.01$ & $17.40 \pm 0.02$ \\
3 & 122144 & 042524 & $17.91 \pm 0.04$ & $18.09 \pm 0.02$ & $17.65 \pm 0.01$ & $17.30 \pm 0.02$ & $16.94 \pm 0.01$ \\
4 & 122148 & 043227 & $18.38 \pm 0.11$ & $17.61 \pm 0.01$ & $16.02 \pm 0.01$ & $15.10 \pm 0.01$ & $14.34 \pm 0.01$ \\
5 & 122150 & 043420 & $19.10 \pm 0.21$ & $18.45 \pm 0.04$ & $17.25 \pm 0.01$ & $16.55 \pm 0.01$ & $15.99 \pm 0.01$ \\
6 & 122157 & 043438 & $16.06 \pm 0.01$ & $15.72 \pm 0.00$ & $14.88 \pm 0.00$ & $14.36 \pm 0.01$ & $13.94 \pm 0.00$ \\
7 & 122204 & 043230 & $16.66 \pm 0.02$ & $16.30 \pm 0.00$ & $15.48 \pm 0.01$ & $15.00 \pm 0.01$ & $14.61 \pm 0.01$ \\
8 & 122205 & 043051 & $18.05 \pm 0.06$ & $18.17 \pm 0.01$ & $17.60 \pm 0.04$ & $17.25 \pm 0.03$ & $16.93 \pm 0.01$ \\
9 & 122143 & 043154 & $19.56 \pm 0.41$ & $19.20 \pm 0.05$ & $17.55 \pm 0.01$ & $16.53 \pm 0.01$ & $15.51 \pm 0.01$ \\
10 & 122133 & 042929 & $19.11 \pm 0.43$ & $18.96 \pm 0.02$ & $18.55 \pm 0.02$ & $18.21 \pm 0.05$ & $17.89 \pm 0.02$ \\
\hline
\end{tabular}

Note. Errors in magnitude represent rms scatter of the night-to-night repeatability over the entire period of SN monitoring. 


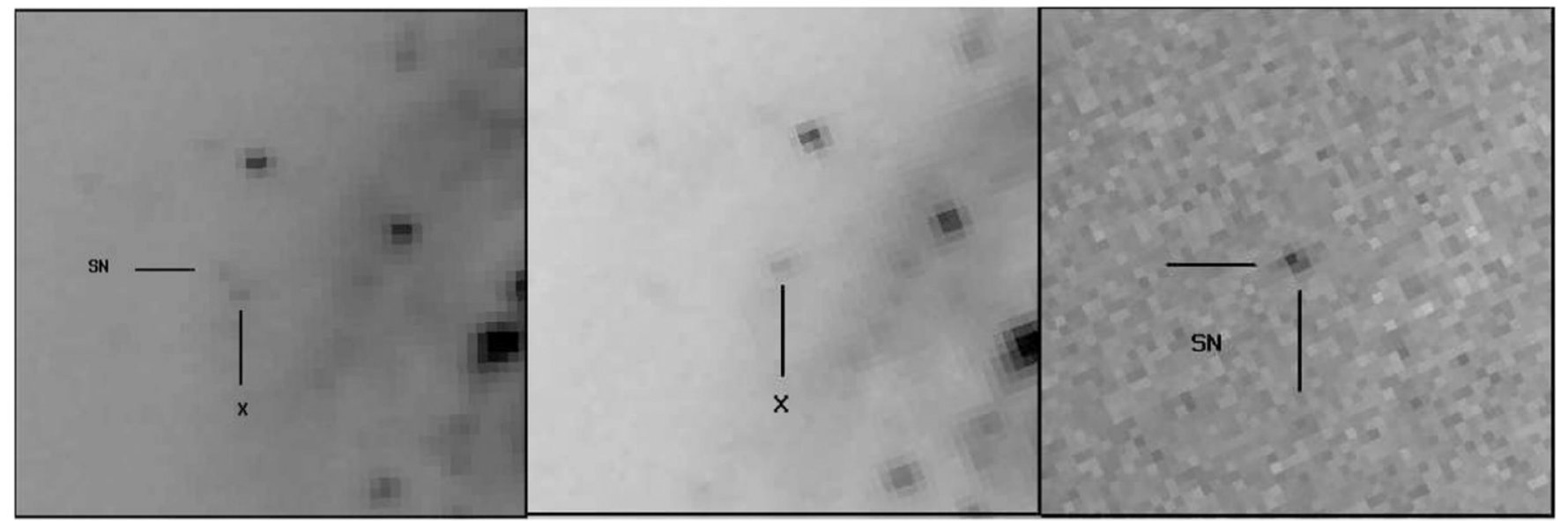

Figure 2. Measurement procedure of the true SN flux in the nebular phase. Each image shows $76^{\prime \prime} \times 76^{\prime \prime}$ around the SN location. North is up and east is to the left. The leftmost panel shows the $V$-band image observed on 2010 January 20 with an SN in it. The middle panel shows the template image taken on 2011 January 4 , with no SN in it. A small flux enhancement marked with a cross (" $\times$ ") symbol is probably due to a star-forming region. The rightmost panel shows the subtracted image, where only an $\mathrm{SN}$ is present.

Table 3

The UBVRI Photometry of SN 2008in

\begin{tabular}{|c|c|c|c|c|c|c|c|c|}
\hline $\begin{array}{l}\text { UT Date } \\
\text { (yy/mm/dd) }\end{array}$ & $\begin{array}{c}\text { JD } \\
2454000+\end{array}$ & $\begin{array}{c}\text { Phase }^{\mathrm{a}} \\
\text { (day) }\end{array}$ & $\begin{array}{c}U \\
(\mathrm{mag})\end{array}$ & $\begin{array}{c}B \\
(\mathrm{mag})\end{array}$ & $\begin{array}{c}V \\
(\mathrm{mag})\end{array}$ & $\begin{array}{c}R \\
(\mathrm{mag})\end{array}$ & $\begin{array}{c}I \\
(\mathrm{mag})\end{array}$ & $\begin{array}{c}\text { Seeing }^{\mathrm{b}} \\
\left({ }^{\prime \prime}\right)\end{array}$ \\
\hline $2008 / 12 / 30.00$ & 830.50 & +5 & $14.23 \pm 0.01$ & $15.33 \pm 0.01$ & $15.07 \pm 0.01$ & $14.86 \pm 0.01$ & $14.86 \pm 0.01$ & 2.9 \\
\hline $2009 / 01 / 01.98$ & 833.48 & +8 & $14.39 \pm 0.01$ & $15.54 \pm 0.01$ & $15.17 \pm 0.01$ & $14.65 \pm 0.01$ & $14.98 \pm 0.01$ & 1.3 \\
\hline 2009/01/09.02 & 840.52 & +15 & $15.47 \pm 0.02$ & $15.76 \pm 0.02$ & $15.21 \pm 0.02$ & $14.89 \pm 0.02$ & $14.87 \pm 0.03$ & 2.5 \\
\hline $2009 / 01 / 23.95$ & 855.45 & +30 & $16.85 \pm 0.04$ & $16.36 \pm 0.01$ & $15.38 \pm 0.02$ & $15.00 \pm 0.01$ & $14.85 \pm 0.01$ & 2.1 \\
\hline $2009 / 01 / 26.02$ & 857.52 & +32 & $16.90 \pm 0.04$ & $16.41 \pm 0.02$ & $15.39 \pm 0.01$ & $14.98 \pm 0.01$ & $14.85 \pm 0.02$ & 3.1 \\
\hline $2009 / 01 / 27.01$ & 858.33 & +33 & $16.81 \pm 0.02$ & $16.41 \pm 0.01$ & $15.39 \pm 0.01$ & $15.00 \pm 0.01$ & $14.83 \pm 0.01$ & 2.8 \\
\hline $2009 / 01 / 28.83$ & 860.33 & & $16.83 \pm 0$ & $16.48 \pm 0$ & $15.43 \pm 0$ & $15.04 \pm$ & & 2.2 \\
\hline $2009 / 01 / 30.88$ & 862.38 & +37 & $17.16 \pm 0.04$ & $16.53 \pm 0.01$ & $15.43 \pm 0.01$ & $15.02 \pm 0.01$ & $14.85 \pm 0.01$ & 2.4 \\
\hline $009 / 02 / 02.89$ & 864.39 & +39 & $17.11 \pm 0.04$ & $16.53 \pm 0.01$ & $15.42 \pm 0.01$ & $14.99 \pm 0.01$ & $14.78 \pm$ & 2.0 \\
\hline $009 / 02 / 15.82$ & 878.32 & +53 & $17.58 \pm 0.05$ & $16.87 \pm 0.01$ & $15.43 \pm 0.02$ & $14.76 \pm 0.01$ & $14.82 \pm$ & 2.8 \\
\hline $2009 / 02 / 16.96$ & 879.46 & +54 & $17.74 \pm 0.06$ & $16.84 \pm 0.01$ & $15.54 \pm 0.01$ & $15.09 \pm 0.02$ & $14.83 \pm 0.01$ & 3.1 \\
\hline 2009/02/17.79 & 880.29 & +55 & $17.79 \pm 0.05$ & $16.87 \pm 0.01$ & $15.55 \pm 0.01$ & $15.10 \pm 0.02$ & $14.85 \pm 0.01$ & 3.1 \\
\hline $2009 / 02 / 20.87$ & 883.37 & +58 & $17.92 \pm 0.07$ & $16.94 \pm 0.01$ & $15.59 \pm 0.02$ & $15.09 \pm 0.01$ & $14.84 \pm 0.01$ & 3.1 \\
\hline $2009 / 02 / 23.77$ & 886.27 & +61 & $18.05 \pm 0.04$ & $17.08 \pm 0.01$ & & $15.13 \pm 0.01$ & & 3.1 \\
\hline $2009 / 02 / 25.81$ & 888.31 & +63 & $18.13 \pm 0.05$ & $17.05 \pm 0.01$ & $15.60 \pm 0.01$ & $15.14 \pm 0.01$ & $14.92 \pm 0.01$ & 2.9 \\
\hline $2009 / 02 / 26.80$ & 889.30 & +64 & & & $15.62 \pm 0.01$ & $15.15 \pm 0.01$ & & 3.8 \\
\hline $2009 / 03 / 02.78$ & 893.28 & +68 & & $17.08 \pm 0.01$ & $15.63 \pm 0.01$ & $15.17 \pm 0.01$ & $14.90 \pm 0.00$ & 4.4 \\
\hline $2009 / 03 / 04.77$ & 895.27 & +70 & & $17.15 \pm 0.01$ & $15.66 \pm 0.01$ & $15.18 \pm 0.01$ & $14.94=$ & 2.4 \\
\hline $2009 / 03 / 09.76$ & 900.27 & +75 & $18.72 \pm 0.14$ & $17.31 \pm 0.02$ & $15.68 \pm 0.01$ & & & 2.3 \\
\hline $2009 / 03 / 15.27$ & 905. & +80 & & $17.20 \pm 0$ & & $15.21 \pm 0.01$ & $14.92 \pm$ & 2.2 \\
\hline$/ 03 / 24.80$ & 915.30 & +90 & $18.73 \pm 0.08$ & $17.42 \pm 0.01$ & $15.94 \pm 0.01$ & $15.48 \pm 0.01$ & $15.15 \pm$ & 2.5 \\
\hline $2009 / 04 / 03.75$ & 925.25 & +100 & $19.28 \pm 0.15$ & $17.66 \pm 0.02$ & $16.10 \pm 0.01$ & $15.57 \pm 0.01$ & $15.31 \pm 0.01$ & 2.2 \\
\hline $2009 / 04 / 05.61$ & 927.22 & +102 & $\ldots$ & & & $15.67 \pm 0.01$ & $15.41 \pm 0.01$ & 2.5 \\
\hline $2009 / 04 / 10.67$ & 932.33 & +107 & $\ldots$ & $18.38 \pm 0.06$ & $16.76 \pm 0.01$ & $16.20 \pm 0.01$ & $15.84 \pm 0.02$ & 2.7 \\
\hline $2009 / 04 / 17.60$ & 939.25 & +114 & $\ldots$ & $19.83 \pm 0.06$ & $17.91 \pm 0.01$ & $17.13 \pm 0.01$ & $16.68 \pm 0.01$ & 2.8 \\
\hline $2009 / 04 / 22.68$ & 944.11 & +118 & & $20.30 \pm 0.16$ & $18.10 \pm 0.03$ & $17.21 \pm 0.03$ & $16.85 \pm 0.04$ & 3.5 \\
\hline $2009 / 04 / 28.71$ & 950.17 & +125 & & $20.40 \pm 0.09$ & 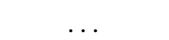 & $17.24 \pm 0.01$ & $16.74 \pm 0.01$ & 2.2 \\
\hline $2009 / 05 / 01.72$ & 953.10 & +127 & $\cdots$ & $20.22 \pm 0.11$ & $18.06 \pm 0.04$ & $17.27 \pm 0.04$ & $16.70 \pm 0.04$ & 2.7 \\
\hline $2009 / 05 / 23.69$ & 975.18 & & & & $18.30 \pm 0.04$ & $17.47 \pm 0.02$ & $17.00 \pm 0.03$ & 2.7 \\
\hline $2009 / 05 / 25.70$ & & & & $20.21 \pm 0.12$ & & $17.45 \pm 0.01$ & & 2.6 \\
\hline $2009 / 05 / 26.71$ & & & & $20.15 \pm 0.17$ & $18.33 \pm 0.04$ & $17.49 \pm 0.02$ & $17.06 \pm 0.03$ & 2.8 \\
\hline $2009 / 06 / 14.69$ & 997.19 & +172 & $\ldots$ & $\ldots$ & $18.39 \pm 0.04$ & & & 2.9 \\
\hline $2010 / 01 / 17.98$ & 1214.49 & +389 & $\ldots$ & $\ldots$ & $\ldots$ & $19.69 \pm 0.07$ & $19.27 \pm 0.13$ & 1.9 \\
\hline $2010 / 01 / 19.91$ & 1216.42 & +391 & $\cdots$ & $21.47 \pm 0.12$ & $20.34 \pm 0.05$ & $19.76 \pm 0.05$ & $19.52 \pm 0.07$ & 2.3 \\
\hline $2010 / 02 / 13.91$ & 1241.42 & +416 & $\ldots$ & $\ldots$ & $20.57 \pm 0.07$ & $19.77 \pm 0.06$ & & 2.2 \\
\hline
\end{tabular}

Notes. The photometric observations are taken with the $1 \mathrm{~m} \mathrm{ST}$, ARIES, Nainital. Errors in magnitude denote $1 \sigma$ uncertainty.

${ }^{a}$ With reference to the explosion epoch JD 2454825.6.

${ }^{\mathrm{b}}$ FWHM of the stellar PSF at the $V$ band. The nights for which we do not have any observation in the $V$ band, PSF is found through interpolation. 
Table 4

The REM Near-infrared Observation of SN 2008in

\begin{tabular}{|c|c|c|c|c|}
\hline $\begin{array}{l}\text { UT Date } \\
(\mathrm{yy} / \mathrm{mm} / \mathrm{dd})\end{array}$ & $\begin{array}{c}\text { JD } \\
2454000+\end{array}$ & $\begin{array}{c}\text { Phase }^{\mathrm{a}} \\
\text { (day) }\end{array}$ & $\begin{array}{c}J \\
(\mathrm{mag})\end{array}$ & $\begin{array}{c}H \\
(\mathrm{mag})\end{array}$ \\
\hline $2008 / 12 / 29.85$ & 830.36 & 4.78 & $14.66 \pm 0.054$ & $14.55 \pm 0.064$ \\
\hline 2009/01/03.87 & 835.37 & 9.78 & $14.60 \pm 0.077$ & $14.38 \pm 0.058$ \\
\hline 2009/01/09.73 & 841.23 & 15.65 & $14.39 \pm 0.059$ & $14.16 \pm 0.072$ \\
\hline $2009 / 01 / 14.73$ & 846.23 & 20.65 & $14.47 \pm 0.107$ & $14.32 \pm 0.150$ \\
\hline 2009/01/19.78 & 851.24 & 25.66 & $14.54 \pm 0.150$ & $\ldots$ \\
\hline 2009/01/24.76 & 856.26 & 30.67 & $14.25 \pm 0.077$ & $14.11 \pm 0.087$ \\
\hline 2009/01/29.87 & 861.37 & 35.79 & $14.37 \pm 0.050$ & $14.14 \pm 0.065$ \\
\hline 2009/02/05.66 & 868.16 & 42.58 & $14.43 \pm 0.067$ & $14.24 \pm 0.107$ \\
\hline 2009/02/11.69 & 874.19 & 48.61 & $14.20 \pm 0.107$ & $14.59 \pm 0.241$ \\
\hline 2009/02/16.72 & 879.22 & 53.64 & $14.43 \pm 0.050$ & $14.35 \pm 0.097$ \\
\hline $2009 / 02 / 21.75$ & 884.25 & 58.67 & $\ldots$ & $14.18 \pm 0.063$ \\
\hline $2009 / 02 / 27.75$ & 890.25 & 64.67 & $\ldots$ & $14.44 \pm 0.077$ \\
\hline 2009/03/04.89 & 895.40 & 69.82 & $14.40 \pm 0.074$ & $14.12 \pm 0.085$ \\
\hline 2009/03/10.74 & 901.24 & 75.66 & $\ldots$ & $14.36 \pm 0.190$ \\
\hline $2009 / 03 / 21.71$ & 912.21 & 86.63 & $\ldots$ & $14.50 \pm 0.090$ \\
\hline $2009 / 03 / 26.73$ & 917.23 & 91.65 & $\ldots$ & $14.41 \pm 0.073$ \\
\hline 2009/04/05.79 & 927.29 & 101.71 & $\ldots$ & $15.00 \pm 0.147$ \\
\hline 2009/04/10.80 & 932.30 & 106.71 & $\ldots$ & $14.94 \pm 0.171$ \\
\hline $2009 / 04 / 15.80$ & 937.30 & 111.72 & $\ldots$ & $14.86 \pm 0.173$ \\
\hline $2009 / 04 / 20.52$ & 942.02 & 116.44 & $\ldots$ & $15.23 \pm 0.232$ \\
\hline
\end{tabular}

Note. ${ }^{\text {a }}$ With reference to the explosion epoch JD 2454825.6.

Table 5

Journal of Spectroscopic Observations of SN 2008in

\begin{tabular}{|c|c|c|c|c|c|c|c|c|c|}
\hline $\begin{array}{l}\text { UT Date } \\
(\mathrm{yy} / \mathrm{mm} / \mathrm{dd} / \mathrm{hh} . \mathrm{hh})\end{array}$ & $\begin{array}{c}\text { JD } \\
2454000+\end{array}$ & $\begin{array}{l}\text { Phase }^{\mathrm{a}} \\
\text { (days) }\end{array}$ & $\begin{array}{l}\text { Range } \\
(\mu \mathrm{m})\end{array}$ & Telescope ${ }^{b}$ & $\begin{array}{c}\text { Grating } \\
\left(\mathrm{gr} \mathrm{mm}^{-1}\right)\end{array}$ & $\begin{array}{l}\text { Slit Width } \\
\left(^{\prime \prime}\right)\end{array}$ & $\begin{array}{l}\text { Dispersion } \\
\left(\AA \text { pixel }^{-1}\right)\end{array}$ & $\begin{array}{c}\text { Exposure } \\
\text { (s) }\end{array}$ & $\begin{array}{c}\mathrm{S} / \mathrm{N}^{\mathrm{c}} \\
\left(\text { pixel }^{-1}\right)\end{array}$ \\
\hline $2008 / 12 / 31 / 22.80$ & 832.45 & +7 & $0.45-1.00$ & HET & 300 & 1.0 & 5.0 & 600 & 45 \\
\hline $2009 / 02 / 17 / 19.74$ & 880.32 & +54 & $0.38-0.68$ & IGO & 600 & 1.5 & 1.4 & 1800 & 34 \\
\hline $2009 / 02 / 22 / 22.50$ & 885.44 & +60 & $0.45-1.00$ & HET & 300 & 1.0 & 5.0 & 600 & 35 \\
\hline $2009 / 04 / 03 / 19.14$ & 925.26 & +99 & $0.38-0.68$ & IGO & 600 & 1.5 & 1.4 & 1800 & 35 \\
\hline $2009 / 04 / 03 / 20.98$ & 925.33 & +99 & $0.60-1.00$ & BTA & 550 & 2.1 & 3.5 & $3 \times 900$ & 60 \\
\hline $2009 / 04 / 22 / 15.49$ & 944.14 & +118 & $0.38-0.68$ & IGO & 600 & 1.5 & 1.4 & 1800 & 11 \\
\hline $2009 / 04 / 23 / 18.00$ & 945.25 & +119 & $0.38-0.79$ & BTA & 600 & 2.1 & 3.5 & $3 \times 900$ & 65 \\
\hline $2009 / 05 / 17 / 02.38$ & 968.10 & +143 & $0.33-0.80$ & NTT & 300 & 1.0 & 4.0 & 2700 & 59 \\
\hline
\end{tabular}

Notes.

${ }^{\text {a }}$ With reference to the explosion epoch JD 2454825.6.

b HET: Low-Resolution Spectrograph on the $9.2 \mathrm{~m}$ Hobby Eberly Telescope; IGO: IFOSC on 2 m IUCAA Girawali Observatory, India; BTA: SCORPIO on the $6 \mathrm{~m}$ Big Telescope Alt-azimuth, Special Astrophysical Observatory, Russia; NTT: EFOSC2 on the $3.6 \mathrm{~m}$ New Technology Telescope, ESO, Chile.

c At $0.6 \mu \mathrm{m}$.

BTA, and NTT, the resolution near $6000 \AA$ is about $10 \AA, 14 \AA$, and $12 \AA$, respectively.

\section{LIGHT-CURVE EVOLUTION}

According to theoretical interpretation, the entire broadband evolution of Type II SNe can be segmented into three phases: the rising phase, the plateau phase, and the nebular phase.

\subsection{The Rising Phase}

The rising phase of the light curve is associated with the shock breakout phenomenon having been theoretically predicted for an SN explosion, in which the radiation-dominated shock wave, generated through the reversal of iron core-collapse, starts to propagate outward through the onion-shell-like layers of the progenitor; when the shock reaches regions with an optical depth of a few tens to unity, the radiation behind the shock escapes the outer surface giving rise to a hot $\left(T>10^{5} \mathrm{~K}\right)$ fireball which emits quasi-blackbody radiation in UV and soft X-rays. This phenomenon is called shock breakout, and depending on envelope mass, density structure, and wind properties of the progenitor, the breakout light curve may last from a few hours to a few days (Grassberg et al. 1971; Chevalier 1976; Falk \& Arnett 1977). Due to the short timescale, the detection of shock breakout is rare, and only recently were the UV light curves of the entire shock breakout phase lasting several hours reported for two Type IIP SNe-SNLS-04D2dc and SNLS-06D1jd (Gezari et al. 2008; Schawinski et al. 2008). Observation of the earliest UV and optical light curves of IIP SNe is crucial to model the shock breakout light curves and constrain the properties of SN 


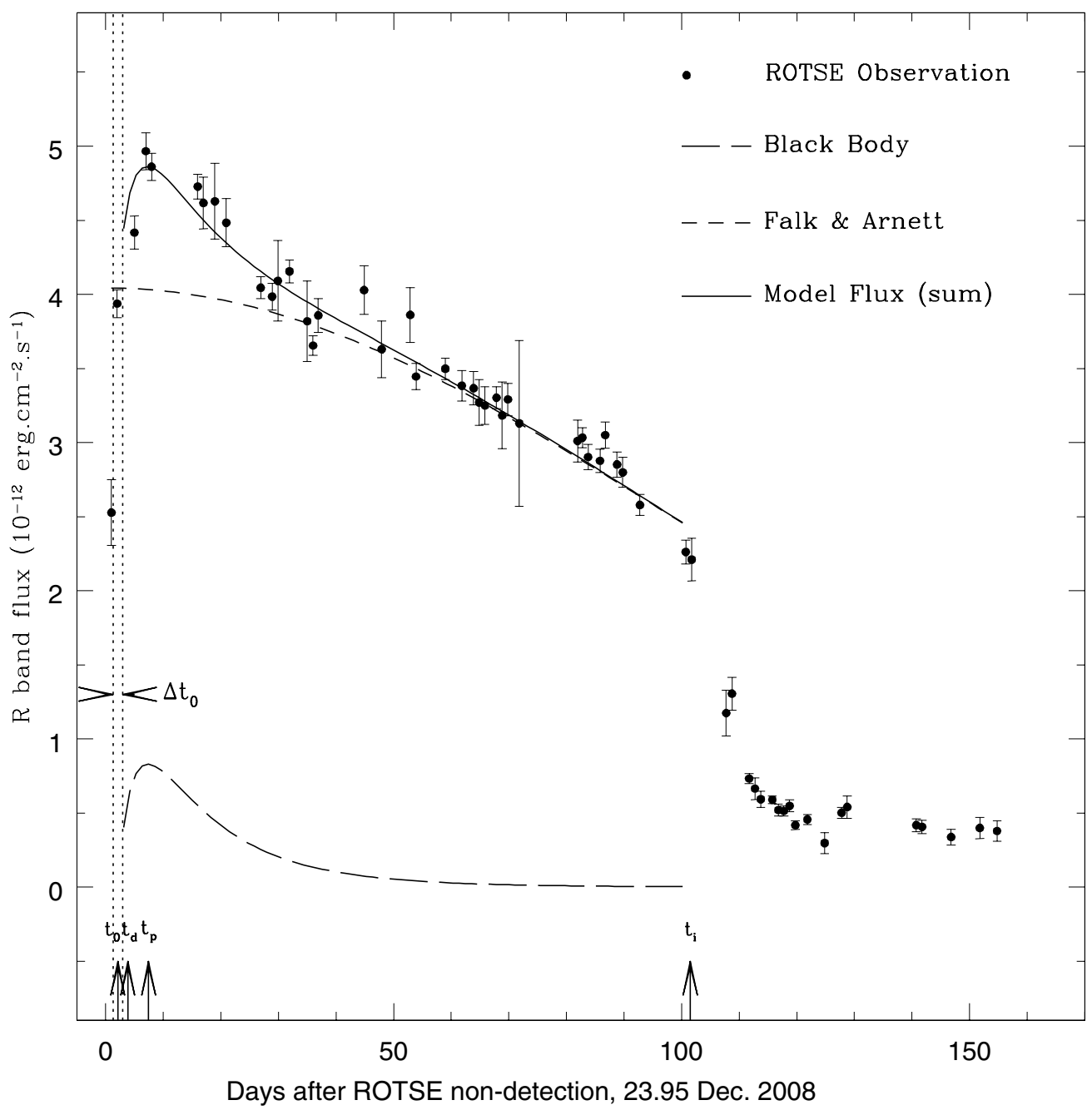

Figure 3. ROTSE $R$-band light curve of SN 2008in. Reference time for the $x$-axis is the epoch of ROTSE non-detection on 2008 December 23.95 . The evolution of the post-shock breakout flux has been modeled by using the simple analytical expressions given in Cowen et al. (2010) and Arnett (1980). The best-fit time for shock breakout is marked with $t_{0}$ while $\Delta t_{0}$ shows the duration of uncertainty. $t_{d}$ is the time of discovery reported by Nakano et al. (2008). $t_{p}$ is the time when $R$ light shows the peak. $t_{i}$ is the time of inflection obtained using the procedure of Elmhamdi et al. (2003a).

progenitors (Tominaga et al. 2009). The earliest SNe IIP optical light curves have been studied in the past for SN 2005cs by Pastorello et al. (2009) and for SN 2006bp by Quimby et al. (2007) and the optical rising phase is rarely observed.

We model the early optical (ROTSE-IIIb $R$-band) light curves of SN 2008in using a simple model and put an observational constraint on the epoch and duration of shock breakout. Following the formulation of Waxman et al. (2007), it can be shown that just after the shock breakout, the intensity of the SN light at a fixed wavelength is proportional to the intensity of blackbody radiation at that wavelength (see Equation (1) of Cowen et al. 2010), while for the plateau phase Arnett (1980) derived an analytical expression (Equation (39) of that paper); hence by combining these two equations one can approximate the overall intensity profile of a Type II SN during the rising phase through the plateau with the following expression:

$$
\begin{aligned}
I_{\mathrm{SN}}(t)= & \frac{A}{\exp \left[B \times\left(t-t_{0}\right)^{0.5}\right]-1} \times\left(t-t_{0}\right)^{1.6} \\
& +C \times \exp \left[-\left\{(t / D)+(t / E)^{2}\right\}\right] .
\end{aligned}
$$

Here, the first term represents the phase associated with the shock breakout while the second term represents the plateau phase. Here $t$ is the time measured in days since 2008 December 23.95 (JD = 2454823.5), the epoch when ROTSE had a nondetection of the SN with limiting magnitude 16.16 in the $R$ band. The time of shock breakout, $t_{0}$, along with the constants $A, B, C, D$, and $E$, are free parameters of the fit. The values of these constants depend on the nature of the progenitor and the kind of explosion. The above model is fitted to the ROTSE data using the $\chi$ minimization technique (Press et al. 1992) and the fit is shown in Figure 3. We obtained a value of the shock breakout time as $t_{0}=2.13 \pm 0.83$ day. This value of $t_{0}$ is consistent with the first data point observed from ROTSE. We shall adopt $t_{0}$ corresponding to JD $=2454825.6$ as the explosion epoch for all the phases of SN 2008in. It is noted that at $V$ and $R$ bands, the SN was detected by Nakano et al. (2008), just 2.4 days after the shock breakout. We note, however, that depending on the extent of the envelope the true core reversal marking the SN explosion would have occurred a few hours earlier (e.g., SN 1987A) or a few days earlier for a red supergiant envelope $\sim 50,000 R_{\odot}$ (Quimby et al. 2007).

In Figure $3, t_{p}(+5.3$ days) corresponds to the peak of the ROTSE $R$-band light curve. A similar peak in the UV light curves of the post-shock breakout phase of the Type IIP supernova SNLS-04D2dc lasting several days was identified as a secondary 


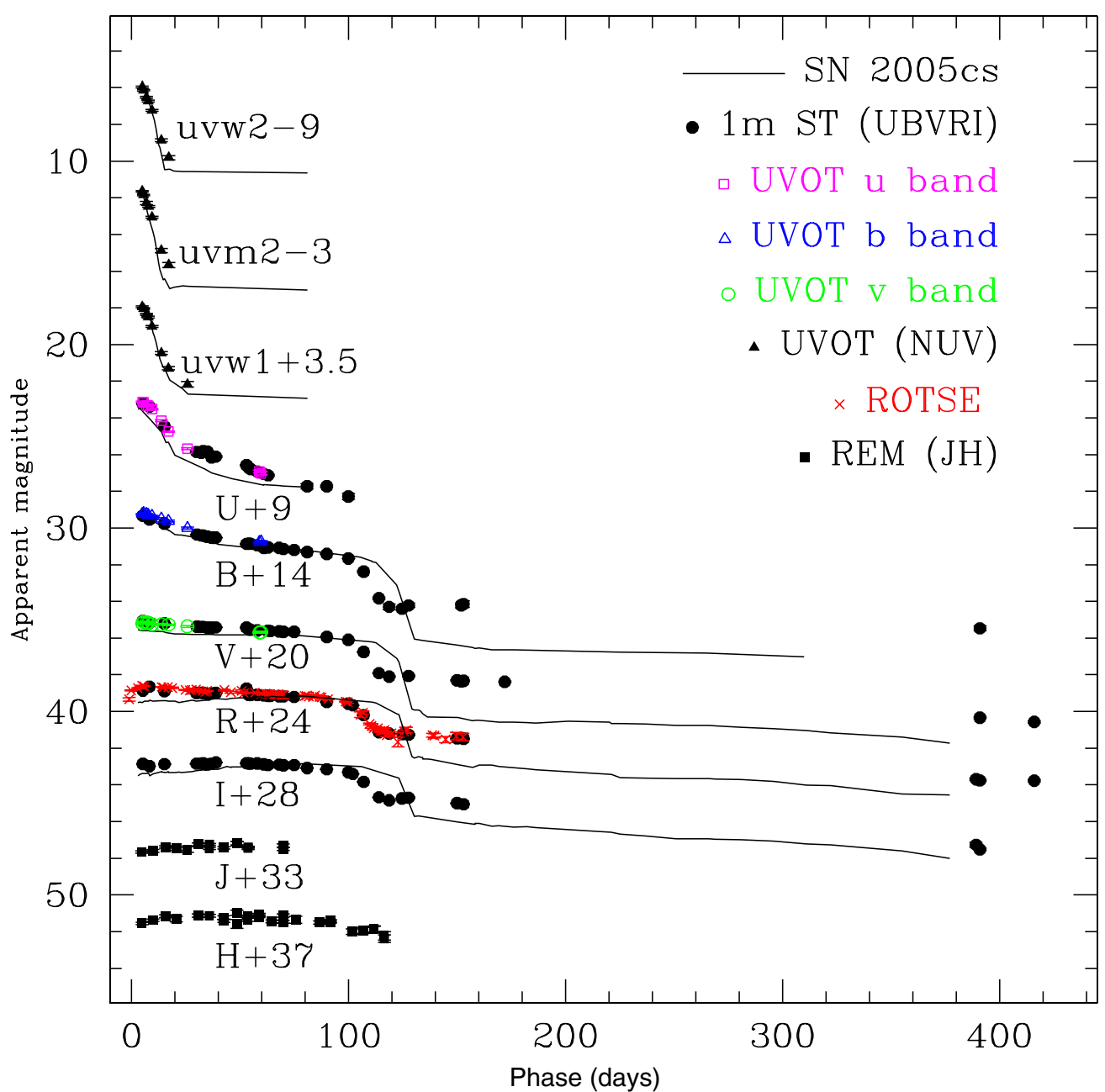

Figure 4. Light curves of SN 2008in in the UV, optical, and NIR bands. The light curves of archetypal low-luminosity SN 2005cs are overplotted for comparison (data are taken from Brown et al. 2009; Pastorello et al. 2009). The light curves of SN 2005cs are scaled in magnitude to match the light curves of SN 2008in.

(A color version of this figure is available in the online journal.)

peak and this peak was explained as the shift of the spectral energy distribution toward longer wavelengths due to the rapid fall in temperature during free adiabatic expansion of optically thick plasma led by a collisionless shock (Schawinski et al. 2008; Tominaga et al. 2009). For SN 2008in we identify the peak in the ROTSE $R$-band curve as a secondary peak and also speculate that for a few other type IIP SNe such as $1999 \mathrm{em}, 1999 \mathrm{gi}$, and $2005 \mathrm{cs}$, where very tiny peaks before the plateau light curve were observed, such features are basically the footprints of the secondary peaks which could be a consequence of shock breakout.

\subsection{The Plateau and Nebular Phase}

The entire UV, optical, and NIR light curves of SN 2008in are shown in Figure 4. For comparison, the light curves of the archetypal low-luminosity SN 2005cs (Brown et al. 2009; Pastorello et al. 2009) are also plotted by scaling them in magnitude to match the observed plateau of SN 2008in. In the early plateau phase, a rapid drop in the UV flux and a slowly declining or constant optical and NIR flux are clearly apparent. The $u v m 2$ magnitude declined from about 14.7 to 18.7 mag within 12 days. The decline rates of the $\mathrm{SN} 2008 \mathrm{in}$ flux in UVOT, $U, B$, and $V$ bands are almost identical with that of SN $2005 \mathrm{cs}$. Starting from the $u v w 2$ until the $R$ band, the measured decline rates from shock breakout to the plateau phase are approximately $0.32,0.34,0.25,0.13,0.04,0.01$, and 0.01 mag day $^{-1}$. The $I J H$ light curves are almost flat even at early phases of the plateau. For this $\mathrm{SN}$, the plateau is well sampled in the BVRI bands, so we can accurately determine the plateau duration. As discussed in Section 3.1, the secondary peak $\left(t_{p} \sim+5.3\right.$ days) in the prompt light curve is generated by the gradually cooling shock-heated SN atmosphere and after that the plateau mechanism starts to dominate. On the other hand, between the plateau and the nebular phase there is another transitional state, when an inflection in the light curve can be seen (Section 7.1). Hence, the plateau duration is precisely the time span between the secondary peak and the inflection. The inflection $\left(t_{i}\right)$ in the $V$-band light curve is observed to occur nearly 103.2 days after the shock breakout. Hence, the time interval between $t_{i}$ and $t_{p}$ is assumed as the duration of the plateau, i.e., $(103.2-5.3) \sim 98$ days.

Once the hydrogen envelope is fully recombined and the ejecta becomes optically thin, the light curve enters the nebular phase and it is sustained mainly by the energy output from radioactive decays of the iron-group elements. During the plateau to nebular transition phase, the $V$-band magnitude drops from $\sim 16.0 \mathrm{mag}$ at around +90 days (still in the plateau) to 18.1 mag at +122 days (in the exponential light-curve tail), i.e., $\sim 2$ mag in about one month. This drop is remarkably smaller than that of SN $2005 \mathrm{cs}$, but consistent with the 2-3 mag drop 


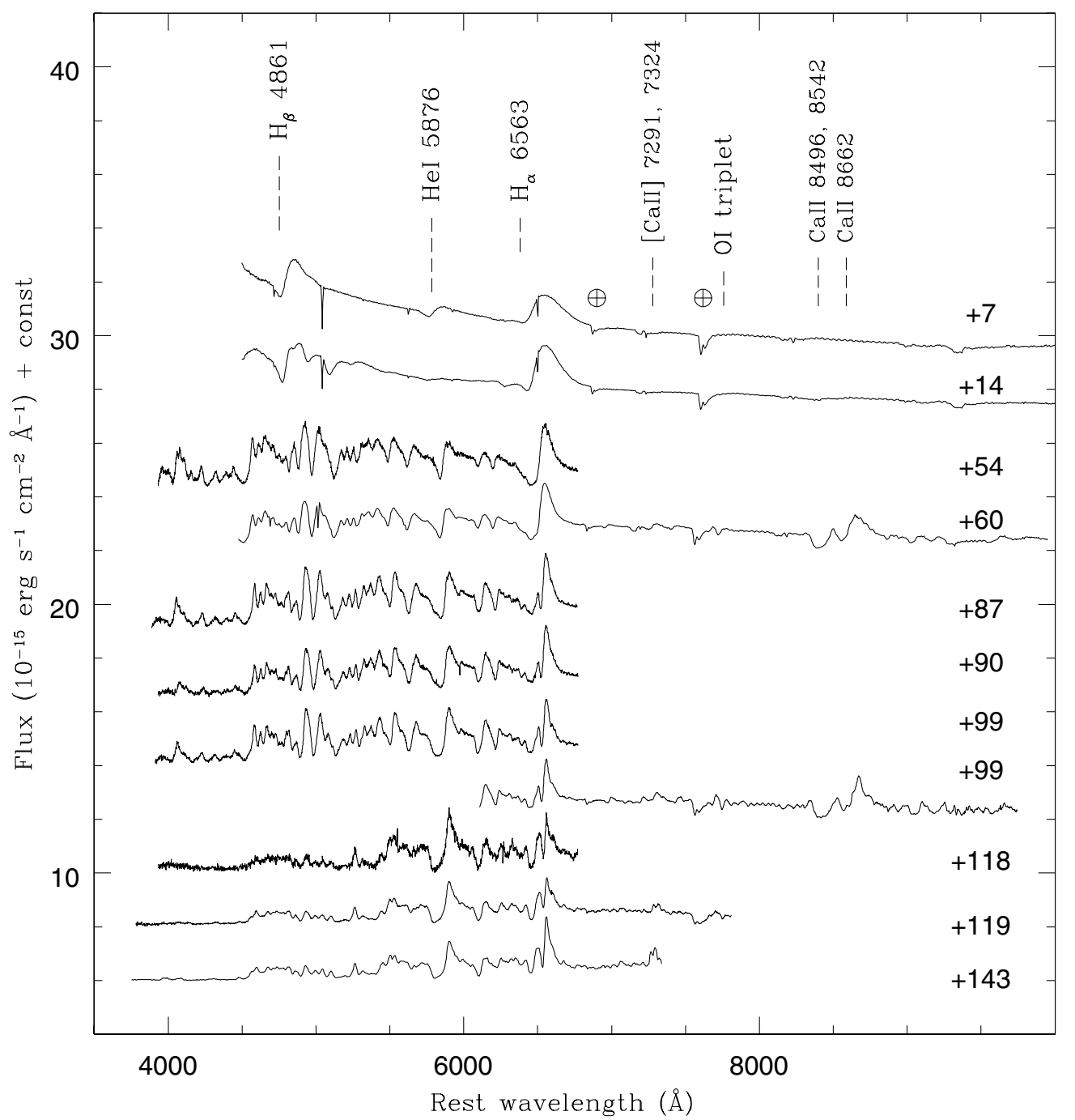

Figure 5. Doppler-corrected flux spectra of SN 2008in observed from +7 days to +143 days. Prominent lines are marked. Telluric features are marked with the $\oplus$ symbol. The sharp absorption dips seen near $5000 \AA$ and $6500 \AA$ in the +7 day and +14 day spectra are artifacts. The spectra signify the early photospheric phase ( +7 days, +14 days), the mid-plateau phase $(+54$ days, +60 days $)$, the late-plateau phase $(+87$ days, +90 days, and +99 days $)$, and the nebular phase $(+118$ days, +119 days, and +143 days).

observed in normal Type IIP SNe (Olivares et al. 2010). A linear fit to the tail from +120 days to +400 days gives the following decline rates (in mag $\left.(100 \text { days })^{-1}\right): \gamma_{B} \sim 0.44, \gamma_{V} \sim 0.84$, $\gamma_{R} \sim 0.91, \gamma_{I} \sim 1.03$ at $B, V, R$, and $I$ bands, which are similar to the values found in normal IIP SNe and comparable with the decay slope of ${ }^{56} \mathrm{Co}$ to ${ }^{56} \mathrm{Fe}$, i.e., $0.98 \mathrm{mag}(100 \text { days })^{-1}$.

\section{SPECTROSCOPIC EVOLUTION}

The spectra of SN 2008in at 10 phases from +7 days to +143 days are shown in Figure 5. The spectra are corrected for the recessional velocity of the host galaxy $(\sim 1567 \pm$ $\left.3 \mathrm{~km} \mathrm{~s}^{-1}\right) .{ }^{23}$ Spectral features are mainly identified as per previously published line identifications for IIP SNe (Leonard et al. 2002; Pastorello et al. 2004). The two earliest spectra ( +7 days and +14 days) show the blue continuum of blackbody emission and have broad P-Cygni profiles of $\mathrm{H} \alpha, \mathrm{H} \beta$, and He I $\lambda 5876$. The next two spectra ( +54 days and +60 days) represent the mid-plateau phase and are marked by a decrease in the continuum and the appearance of more P-Cygni profiles

\footnotetext{
${ }^{23}$ http://leda.univ-lyon1.fr/
}

for the permitted metallic (Fe II, Sc II, Ti II, Ba II, and Mg II), OI $\lambda 7773$, Na I D, and Ca II IR triplet lines similar to other normal Type IIP SNe (see Roy et al. 2011 and references therein). The spectra at phases +87 days, +90 days, and +99 days represent the end stages of the plateau and these are marked by a redder continuum and decreasing line widths of hydrogen lines. The +118 day, +119 day, and +143 day spectra represent the nebular phase having negligible continuum and are marked by pronounced emissions of forbidden lines [Ca II] $\lambda \lambda 7291,7323$, and fading of the absorption features of hydrogen and metallic lines.

In Figure 6, we compare the +99 day spectrum of SN 2008in with the spectra of other Type IIP SNe observed at similar phases that is at roughly the time when the light curve changes from the photospheric plateau to the nebular phase. The phases quoted for each event are with reference to the moment of inflection $\left(t_{i}\right.$; see Figure 3). The narrow P-Cygni $\mathrm{H} \alpha$ profile and the presence of strong lines of Ba II at $\lambda 6142$ and $\lambda 6497$ of SN 2008in show striking resemblance to the low-luminosity SNe 1997D, 1999br, $1999 \mathrm{eu}, 2001 \mathrm{dc}$, and $2005 \mathrm{cs}$. On the other hand, the normal Type IIP SNe 2004et and 2008gz show broader profiles of $\mathrm{H} \alpha$ and weaker lines of Ba II. It is noted that in the blue wing of $\mathrm{H} \alpha$ 


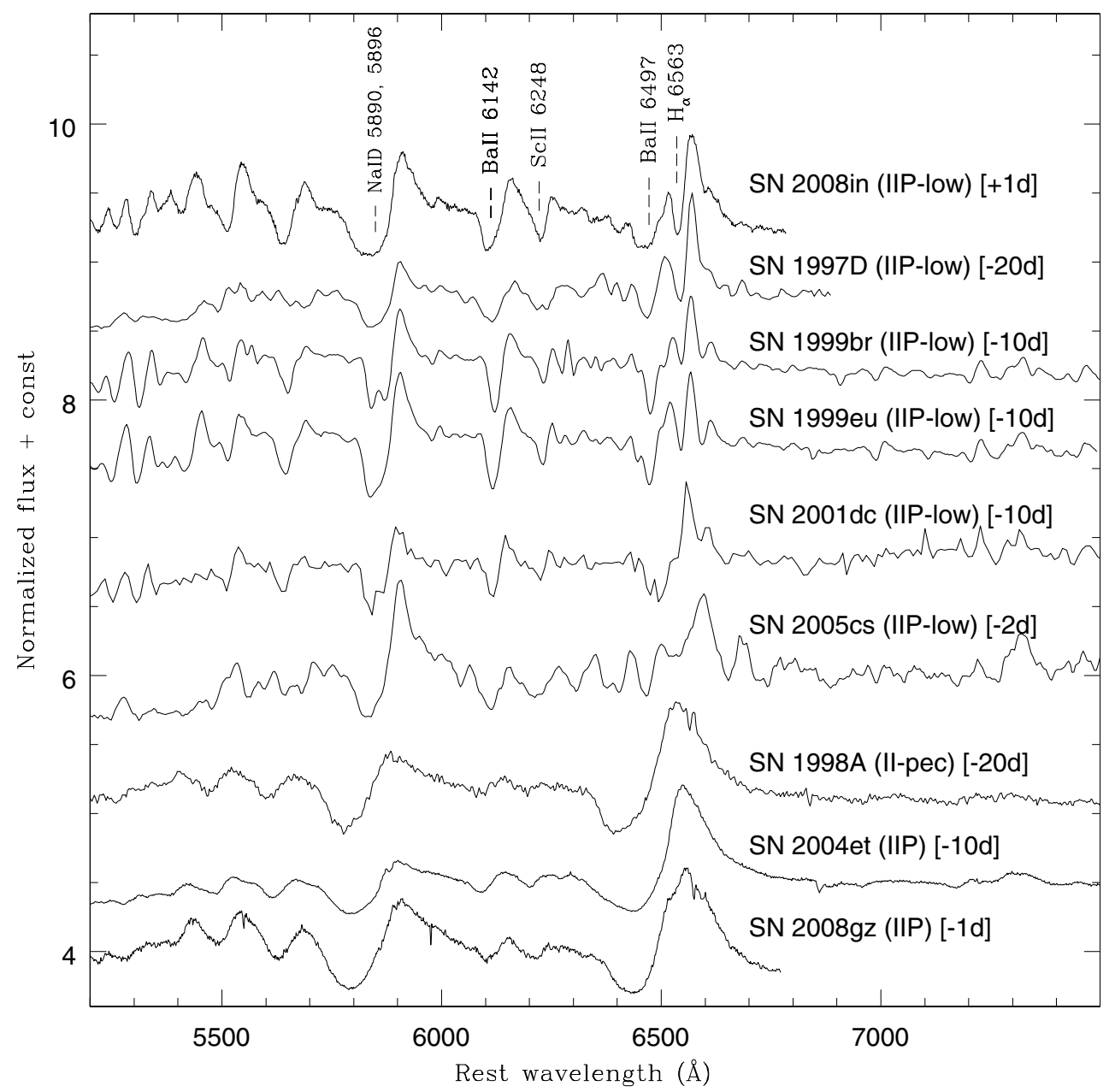

Figure 6. End plateau spectrum (+99 days) of SN 2008in is compared with five low-luminosity SNe 1997D, 1999br, 1999eu, 2001dc, and 2005cs (Pastorello et al. 2009 and references therein), two normal SNe 2004et (Misra et al. 2007) and 2008gz (Roy et al. 2011), and a peculiar Type II SN 1998A (Pastorello et al. 2005), observed at comparable epochs. The phases quoted for each event are with reference to the moment of inflection $\left(t_{i}\right.$; see Figure 3$)$, which marks the highest rate of decline in the $V$-band light curve during the end of the plateau and the beginning of the nebular phase (Elmhamdi et al. 2003a). All the spectra have low spectral resolution ( $\sim 10 \AA)$. The P-Cygni features of Na I D, Ba II $\lambda 6142$, Sc II $\lambda 6248$, Ba II $\lambda 6497$, and $\mathrm{H} \alpha$ are marked.

more metallic lines get resolved than in the normal IIP SNe and this arises due to smaller line widths of hydrogen lines seen in low-luminosity IIP SNe (Fraser et al. 2010 and references therein). In addition, the $\mathrm{Ba}$ II line in the low-luminosity $\mathrm{SNe}$ shows stronger absorption components due to lower ejecta temperature than that in the normal luminosity Type IIP SNe 2004et and 2008gz (Turatto et al. 1998). In Figure 7, we have identified the spectral features in a late-plateau phase (+99 days) spectrum which covers the full wavelength range from 0.4 to $0.95 \mu \mathrm{m}$. For line identification we have followed Pastorello et al. (2004), where different spectral lines were identified for the +102 day spectrum of a low-luminosity SN 1999br. We are able to identify all the features and the spectral profiles of all the elements are similar to the archetypal low-luminosity IIP SNe 1997D, 2005cs, and 1999br (Pastorello et al. 2004 and references therein).

Figure 8 shows the spectral evolution of $\mathrm{H} \alpha, \mathrm{H} \beta$, Na I D, Fe II, Ba II, and Sc II lines. In Figure 9 (left panel), we show the expansion velocities of the ejecta derived from Balmer and $\mathrm{Fe}$ II lines. The latter is a good indicator of photospheric velocity. The expansion velocities of the $\mathrm{H}$-envelope are estimated using absorption minima of the P-Cygni profiles and at the two earliest phases ( +7 days and +14 days) $\mathrm{H} \alpha$ and $\mathrm{H} \beta$ show a broad P-Cygni profile which, with time, becomes narrower at later phases keeping the position of the emission peak centered near zero velocity. The $\mathrm{H} \alpha$ line velocity starts at about $7000 \mathrm{~km} \mathrm{~s}^{-1}$ at +10 days, reaches $4000 \mathrm{~km} \mathrm{~s}^{-1}$ at +50 days, and flattens at a level of $1200 \mathrm{~km} \mathrm{~s}^{-1}$ in the nebular phases. It can be seen from the right panel of Figure 9 that in the comparable phases the $\mathrm{H} \alpha$ line velocities of low-luminosity $\mathrm{SNe}$ are less than half those of normal IIP SNe 1999em and 2004et, whereas the SN 2008in velocities are more like low-luminosity SN $2005 \mathrm{cs}$. To estimate the photospheric velocity of the transient, we have computed the velocities of different Fe II lines $\lambda 4924, \lambda 5018$, and $\lambda 5169$ at different phases. The first marginal detection of these lines is in the +14 day spectrum and they became prominent in later stages of evolution. For SN 2008in, the average velocity of these lines (and hence roughly the photospheric velocity) at +14 days is about $4450 \mathrm{~km} \mathrm{~s}^{-1}$, which is comparable with that of lowluminosity SNe and much less than ordinary Type IIP events (see Figure 12 of Pastorello et al. 2009).

The emergence of absorption dips in the blue wings of $\mathrm{H} \alpha$ is clearly seen in the post +60 day spectra ${ }^{24}$ and the absorption dips have been marked with $\mathrm{A}, \mathrm{B}$, and $\mathrm{C}$ in Figure 8. We distinguish "A" as an absorption dip due to Ti II and $\mathrm{H} \alpha$ and

\footnotetext{
${ }^{24}$ This is the first time that the rapidly changing evolution of $\mathrm{H} \alpha$ profiles during the late plateau to nebular phase has been so densely covered in a low-luminosity SN.
} 


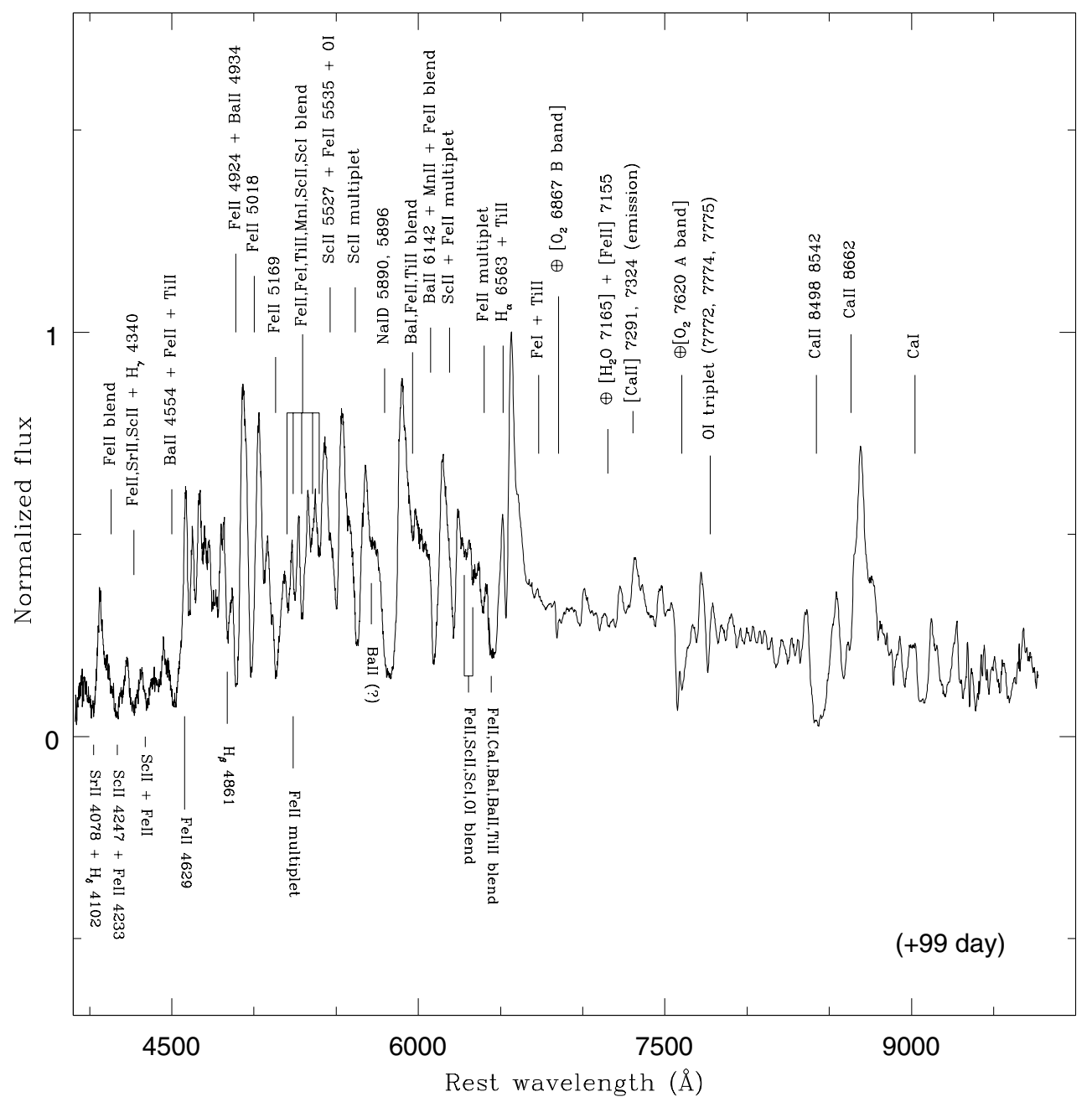

Figure 7. Spectral line identifications in a late-plateau phase (+99 days) spectrum are shown. Spectral features are mainly identified as per previously published line identifications for IIP SNe (Leonard et al. 2002; Pastorello et al. 2004). Telluric features are marked with the " $\oplus$ " symbol.

its equivalent width (EW) increases from $12.85 \AA$ at +60 days to $19.45 \AA$ at +143 days. This progressively stronger absorption dip requires a steep deceleration of the $\mathrm{H} \alpha$ emitting zone along with its depletion by inner metal shells (like Ti II). The broader absorption dip "B" is identified as a blend of Ba II $\lambda 6497$, Bar, $\mathrm{Ca}$ I, and Ti II. An exceptionally strong Ba II line is quite typical for cooler ejecta of less energetic faint SNe. The segment " $\mathrm{C}$ " is speculated to be a footprint of $\mathrm{Fe}$ II multiplets as the evolution of its linewidths seems to be correlated with the other Fe II lines. It is noted, however, that in a few normal Type IIP SNe 1999em, 2004et, and 2008gz, the spectral feature near "C" at early plateau phases was also identified as a signature of a high-velocity $\mathrm{H} \alpha$ component, and in the present spectra its presence cannot be completely ruled out.

The evolution of $\mathrm{H} \beta$ is similar to the evolution of $\mathrm{H} \alpha$. The $\mathrm{P}$-Cygni profile of $\mathrm{H} \beta$ is clearly visible in the +7 day spectrum, and later on, the emission component is blanketed by various metal lines, mainly due to $\mathrm{Fe}$ II $\lambda 4924, \lambda 5018$, and $\lambda 5169$. Traces of $\mathrm{H} \beta$ and $\mathrm{Fe}$ II absorption dips indicate that from the beginning of the nebular phase $\mathrm{H}$ shells of ejecta and the regions containing Fe II ions move with a comparable velocity, reaching an asymptotic value of $\sim 1000 \mathrm{~km} \mathrm{~s}^{-1}$ nearly 140 days after the explosion. In the +14 day spectrum, Fe II lines are marginally detected while in the spectra between +60 days and +99 days they are prominent. We investigated the temporal change of EW of a relatively less blended Fe II $\lambda 4924$; from the spectra normalized to the $\mathrm{H} \alpha$ peak value we found an $\mathrm{EW} \sim 0.67 \AA$ at +14 days, increasing to $17.45 \AA$ at +54 days and to $26.53 \AA$ in the +99 day spectrum. Later on, the EW decreased to $19.07 \AA$ in the +118 day spectrum and finally reached $18.27 \AA$ in the +143 day spectrum. This rapid fall in the EW of inner metal-rich shells plausibly indicates a decrease in the opacity of the SN inner ejecta.

Traces of the He I $\lambda 5876$ line are also seen in the +7 day spectrum (Figure 8). The ratio of EWs between $\mathrm{H} \alpha$ and $\mathrm{He}$ I for this spectrum is $\mathrm{EW}(\mathrm{H} \alpha) / \mathrm{EW}(\mathrm{He} \mathrm{I}) \sim 1.99$. In the +14 day spectrum, this ratio has increased to 5.36. The steep decrement in EW of He I seems to be due to a quick recombination of $\mathrm{He} \mathrm{I}$ ions as a result of the rapid fall in temperature of the constantly rarefying ejecta. From +54 days, the emerging P-Cygni feature of Na I D becomes prominent. This feature seems to be a perfect P-Cygni throughout our entire spectral sequence. It simply indicates a uniform spherical distribution of Na I in the SN ejecta. In the three high $\mathrm{S} / \mathrm{N}$ spectra labeled with +54 days, +60 days, and +87 days, a tiny absorption dip overlaid on the emission component of the Na I D P-Cygni is seen, and since these spectra are redshift corrected, these dips are impressions of intervening interstellar matter present in the host galaxy. The absence of any similar absorption dip in the blue wing of the Na I D profile, expected from Galactic interstellar matter, confirms that there is little absorption from the Milky Way in the line of sight of SN 2008in. 


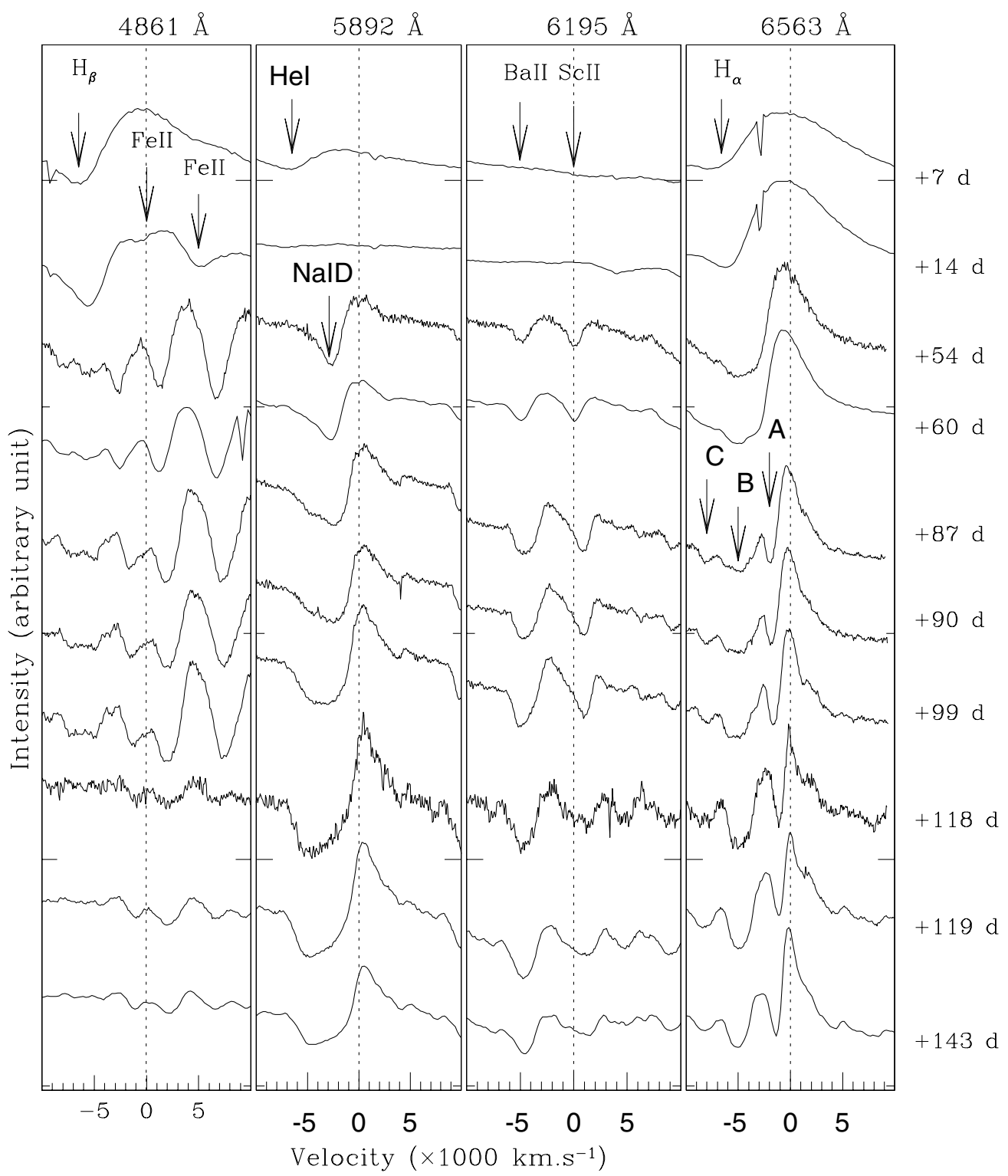

Figure 8. Spectral evolution of $\mathrm{H} \beta$, Fe II $\lambda 4924$, Fe II $\lambda 5018$, He I $\lambda 5876$, Na I D $\lambda \lambda 5890,5896$, Ba II $\lambda 6148$, Sc II $\lambda 6248$, Ba II $\lambda 6497$, and $\mathrm{H} \alpha$ lines of SN 2008 in during its transition from the plateau to the nebular phase. The dotted line at zero velocity corresponds to the rest wavelength, marked at the top of each panel. The sharp absorption dips seen in the $\mathrm{H} \alpha$ profile for +7 day and +14 day spectra are artifacts.

The spectral evolution of Sc II $\lambda 6248$ and the s-process element Ba II $\lambda 6142$ is clearly seen in SN 2008in. In the +7 day and +14 day spectra, there is no clear evidence for the presence of these two elements; however, in the +54 day spectrum they are prominent. The absorption components become stronger with time and persist until the +143 day spectrum. In contrast, in the normal Type IIP SNe 2004et (Sahu et al. 2006), 1999em (Elmhamdi et al. 2003b), and 2008gz (Roy et al. 2011) these features disappeared at $\sim 170$ days after the explosion. The ejecta of underluminous Type IIP SNe expand at a lower velocity than those of normal ones. So, the Ba lines in low-luminosity events persist for a longer time just because the ejecta takes more time to cool down. As a result, it takes longer to become optically thin.

In the nebular phase, the forbidden lines [Ca II] $\lambda \lambda 7291,7323$, [O I] $\lambda \lambda 6300,6364$, and [Fe II] $\lambda 7155$ are among the strongest features visible in the spectra. Also, the line ratios of emission lines of $\mathrm{Ca}$ II and the $\mathrm{O}$ I doublet in late nebular phases are a good indicator of the progenitor mass (Fransson \& Chevalier
1987). In Figure 10, we compare the +139 day and +283 day spectra of SN 19997D (Benetti et al. 2001) with the nebular phase spectra of SN 2008in. The [Ca II] doublet is seen in the +99 day, +119 day, and +143 day spectra of SN 2008in, whereas a small footprint of the O I doublet can be seen in the +143 day spectrum. The [Fe II] line is not seen in our spectra. Considering that the explosion epoch of SN 1997D was quite uncertain, it is likely that the spectral evolution of $\mathrm{H} \alpha$ and forbidden lines of SN 2008in is quite similar to that of SN 1997D.

\section{EXTINCTION AND DISTANCE TOWARD SN 2008in}

In order to determine the bolometric light curve and other physical parameters, a correct estimate of the extinction and distance toward the SN is essential. We adopt the Galactic reddening along the line of sight of SN 2008in as derived from the $100 \mu \mathrm{m}$ all sky dust extinction map (Schlegel et al. 1998), i.e., $E(B-V)=0.0224 \pm 0.0003 \mathrm{mag}$. In order to estimate reddening due to the host galaxy M61, we used the 

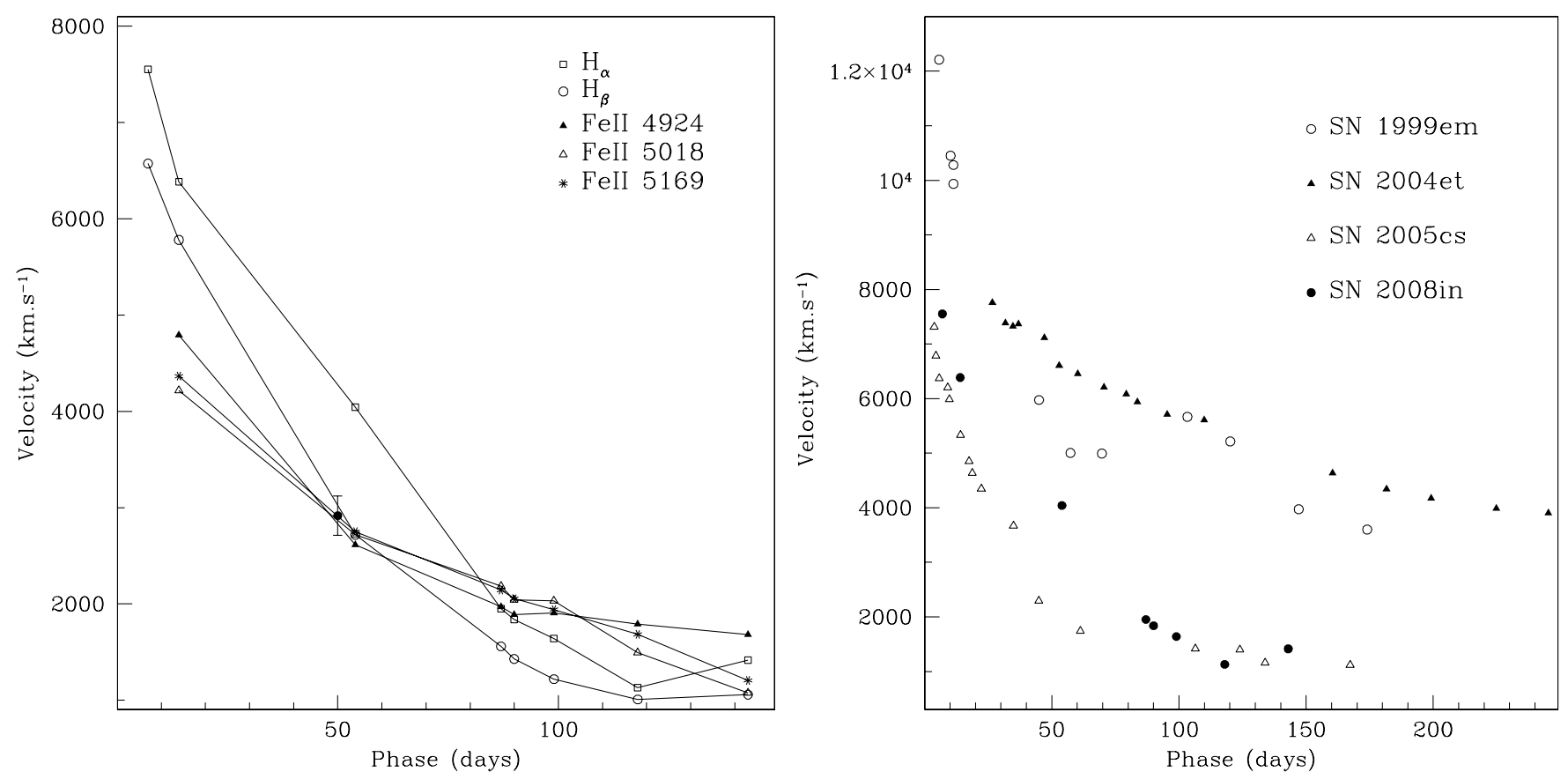

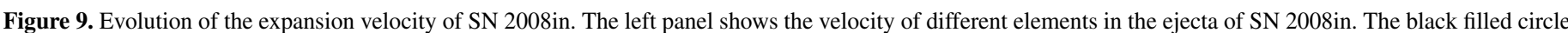

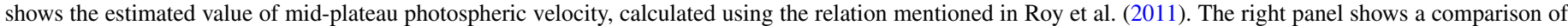

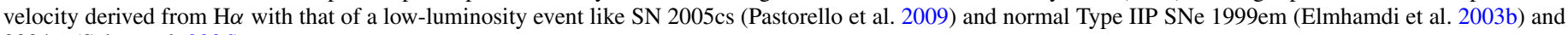
2004et (Sahu et al. 2006).

spectrum of SN 2008in taken on 2009 February 17 from the $2 \mathrm{~m}$ IGO telescope having good $\mathrm{S} / \mathrm{N}(\sim 40)$ and corrected for mean heliocentric radial velocity of the host $\left(c z \approx 1567 \pm 3 \mathrm{~km} \mathrm{~s}^{-1}\right)$. Near the zero velocity, the spectrum showed a tiny absorption feature overlaid on the emission component of the P-Cygni profile of $\mathrm{Na}$ I D (Section 4). The EW of this absorption feature was computed as $0.535 \pm 0.713 \AA$. The error in EW is estimated using Equation (6) of Vollmann \& Eversberg (2006). It is known that the EW of the interstellar absorption bands is well correlated with the reddening $E(B-V)$ estimated from the tail of the SN Ia color curve (Barbon et al. 1990; Richmond et al. 1994; Turatto et al. 2003), and hence using the empirical relation $E(B-V)=$ $-0.01+0.16 \mathrm{EW}$ (EW in $\AA$ ), given by Turatto et al. (2003), we obtain the host contribution as $E(B-V) \approx 0.076 \pm 0.104$, which is considerably higher than the Galactic contribution. This is consistent with the absence of any absorption feature due to Galactic interstellar matter in the blue wing of the emission component of the $\mathrm{Na}$ I D profile. Consequently, we adopt an $E(B-V)$ (estimated as a sum of Galactic and host galaxy reddening) of $0.0984 \pm 0.104 \mathrm{mag}$ for SN 2008in. This corresponds to a visual extinction $\left(A_{V}\right)$ of $0.305 \pm 0.322$. Considering the uncertainty in the $E(B-V)$ estimated using $\mathrm{Na}$ I D lines, a lower value of $E(B-V)$ of $0.0448 \pm 0.0006 \mathrm{mag}$ (twice the Galactic reddening) and the corresponding $A_{V}$ of $0.139 \pm 0.002$ cannot be ruled out. We will discuss its implication for the derived properties of the SN.

The Hubble flow distance of the host galaxy M61, after correction for the Virgo infall, is estimated as $13.7 \pm 1.1 \mathrm{Mpc}^{25}$ The distance estimated through the Tully-Fisher method is $12.1 \pm 2.7 \mathrm{Mpc}^{26}$ Additionally, we also calculated the distance following the Standard Candle Method for Type II SNe

\footnotetext{
25 The cosmological model with $H_{0}=70 \mathrm{~km} \mathrm{~s}^{-1} \mathrm{Mpc}^{-1}, \Omega_{m}=0.3$, and $\Omega_{\Lambda}=0.7$ is assumed throughout the paper and the uncertainty corresponds to a local cosmic thermal velocity of $208 \mathrm{~km} \mathrm{~s}^{-1}$ (Terry et al. 2002).

26 http://nedwww.ipac.caltech.edu/
}

(Hamuy \& Pinto 2002; Hamuy 2005; Hendry et al. 2005). It is found that there is a strong correlation between the distance of Type IIP SNe along with their mid-plateau apparent $V$ - and $I$-band magnitudes and the mid-plateau photospheric velocity, for a given cosmological model. For SN 2008in we estimate the mid-plateau $(\sim+50$ days $)$ apparent magnitudes $\sim 15.56 \pm 0.05 \mathrm{mag}$ for $V$ and $\sim 14.77 \pm 0.06 \mathrm{mag}$ for the $I$ band. Considering the $V$ - and $I$-band extinctions toward the $\mathrm{SN}$, $0.305 \pm 0.322 \mathrm{mag}$ and $0.183 \pm 0.193 \mathrm{mag}$, respectively, we derive a distance of $\sim 12.23 \pm 1.87 \mathrm{Mpc}$. The adopted value of mid-plateau photospheric velocity is $2694.67 \pm 70 \mathrm{~km} \mathrm{~s}^{-1}$ (see Section 7.2). Combining the above three measurements, we adopt the weighted mean distance of $13.19 \pm 1.09 \mathrm{Mpc}$, which corresponds to a distance modulus of $30.6 \pm 0.2$.

\section{COLOR EVOLUTION AND BOLOMETRIC FLUX}

Figure 11 shows the temporal evolution of the reddeningcorrected broadband colors of SN 2008in. For comparison, we overplot the color curves for well-studied SNe 1987A (Suntzeff \& Bouchet 1990), 1999em (Elmhamdi et al. 2003b), 2004et (Sahu et al. 2006; Maguire et al. 2010), 2005cs (Pastorello et al. 2009), and SN 2008gz (Roy et al. 2011). After small differences during the initial phases, the plateau phase color evolution of all Type II SNe is more or less similar. The $(U-B)_{0}$ and $(B-V)_{0}$ colors are blue during early photospheric phases and they become redder by about 1-2 mag in the plateau phase while the $(V-R)_{0}$ and $(V-I)_{0}$ colors evolve slowly and become red only by about $0.5 \mathrm{mag}$. The $(J-H)_{0}$ color remains constant at $\sim 0.25$ mag. During the transition phase from plateau to nebular, the low-luminosity SN 2005cs showed a striking red peak in the $(B-V)_{0},(V-R)_{0}$, and $(V-I)_{0}$ colors. For SN 2008in, this red peak is not present and its color evolution is found to be consistent with the normal Type IIP SNe.

The quasi-bolometric light curve estimated from the UV, optical, and IR broadband (UVOIR) magnitudes of SN 2008in 


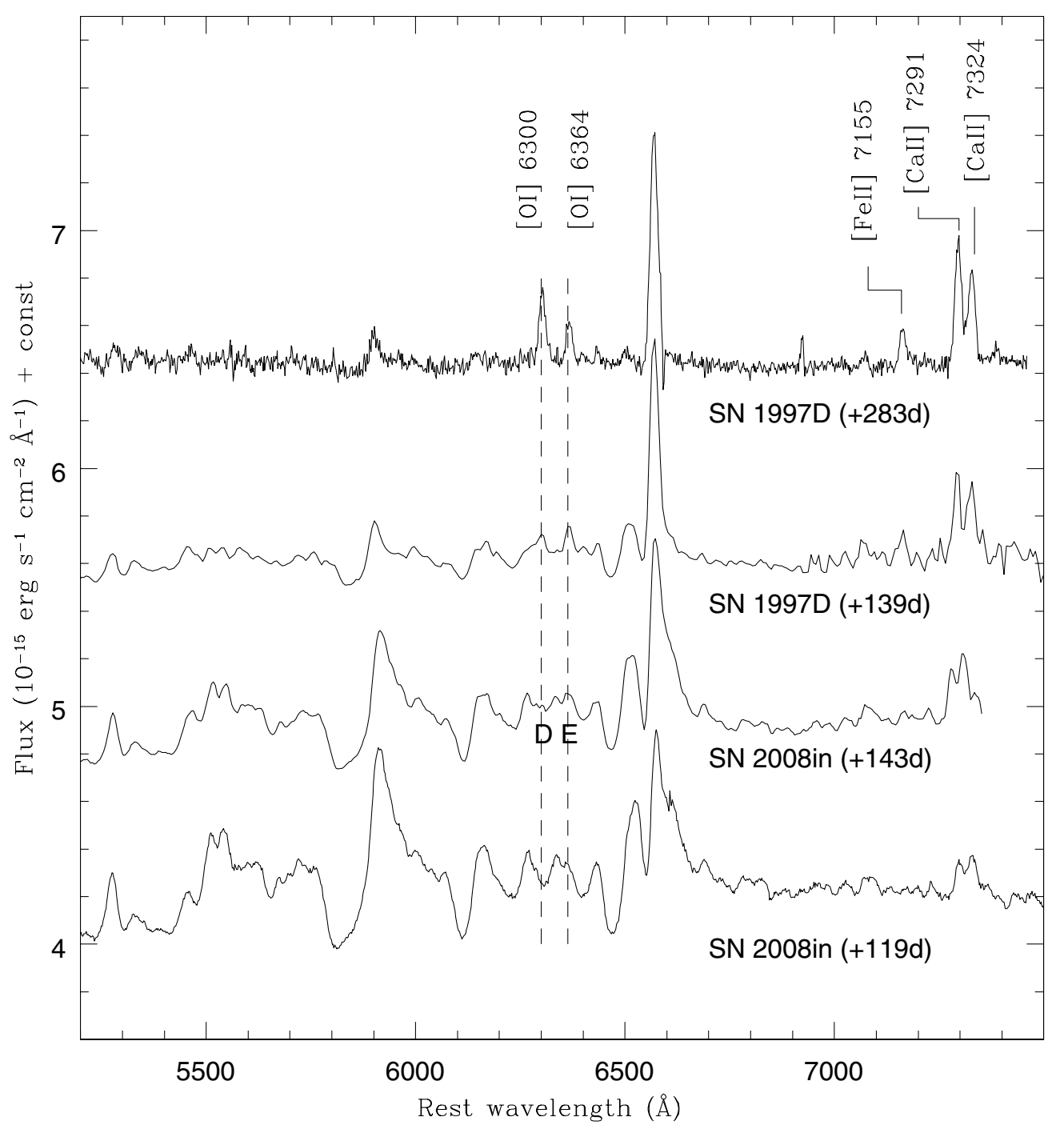

Figure 10. Forbidden lines during the nebular phases of SN 2008in are compared with the low-luminosity SN 1997D. The lines [O I], [Fe II], and [Ca II] are marked.

is shown in Figure 12 along with those of other Type II SNe. The extinction-corrected magnitudes are first converted into fluxes using zero points given by Bessell et al. (1998) and then the total flux in the UVOIR bands is obtained after a linear interpolation and integration between 0.203 and $1.67 \mu \mathrm{m}$. The fluxes were calculated on those nights for which we had complete observations in UBVRI bands. For the initial two weeks the SN was detected by Swift/UVOT in near-ultraviolet bands with a significant flux density. This contribution has been accounted for while measuring the net $U V O I R$ flux. It has been assumed that contribution in the $U$ band is mainly important during the plateau and decreases rapidly to about 5\% in the nebular phase (see Misra et al. 2007; Roy et al. 2011 and references therein). From the light curve it is noticeable that the near-ultraviolet flux is almost negligible beyond 20 days after explosion. The $J H$ contribution during the plateau is calculated from our data, whereas for the nebular phase, due to lack of data, we are not able to make any direct measurement. For most low-luminosity SNe IIP, the NIR flux contribution in the nebular phase is about 50\% of the total flux (Pastorello et al. 2009). We have therefore increased the net flux by 55\% to account for the maximal contributions from the $U$ and NIR bands at phases later than +140 days. Figure 6 and 12 clearly demonstrate that spectroscopically SN 2008in appears to be like low-luminosity
SNe IIP, but photometrically it appears quite normal. In the following section, the UVOIR curve is used to estimate the amount of radioactive ${ }^{56} \mathrm{Ni}$ and other physical parameters that characterize the explosion and the progenitor star.

\section{PHYSICAL PARAMETERS}

The physical entities that seem to govern the entire scenario are mainly associated with the nature of the progenitor and the radioactive elements (mainly ${ }^{56} \mathrm{Ni}$ ) generated inside the inner portion of the ejecta during the explosion. ${ }^{56} \mathrm{Ni}$ is synthesized by the explosive burning of $\mathrm{Si}$ and $\mathrm{O}$ during the shock breakout (Arnett 1980, 1996). Over time this material is eventually converted to ${ }^{56} \mathrm{Co}$ and then to ${ }^{56} \mathrm{Fe}$ by means of radioactive transitions having decay times of 8.77 and 111.3 days, respectively. The $\gamma$-rays and neutrinos emitted during this process sustain the nebular phase light curve, and consequently the observed tail luminosity becomes a good tracer for the ejected synthesized ${ }^{56} \mathrm{Ni}$.

\subsection{Produced Radioactive Nickel}

We use different methods to estimate mass of ${ }^{56} \mathrm{Ni}$. Hamuy (2003) proposed a relation between bolometric tail luminosity and the synthesized ${ }^{56} \mathrm{Ni}$ during core-collapse $\mathrm{SNe}$ by 


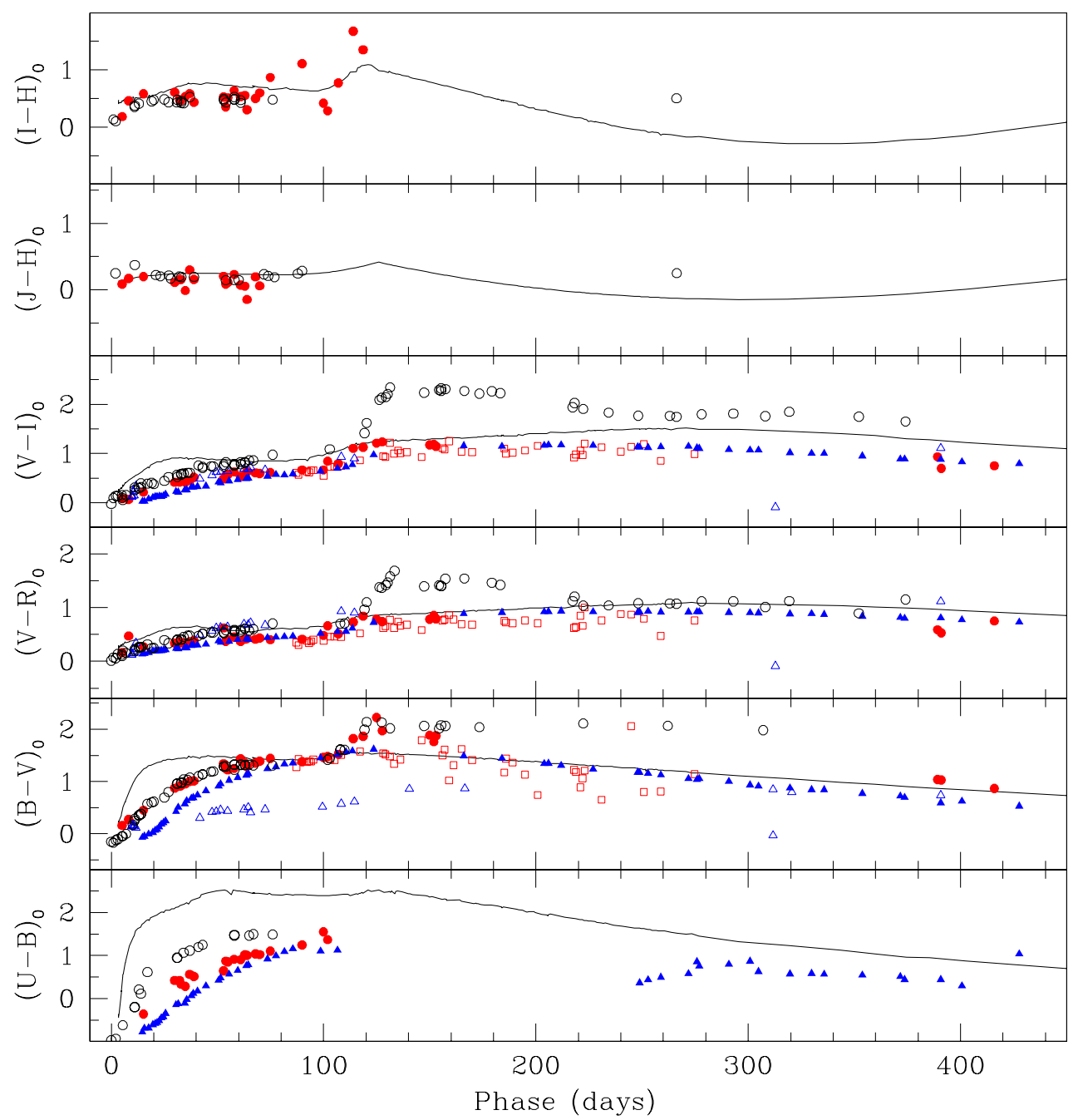

Figure 11. Color curve of SN 2008in is shown with filled circles. Also shown are the SNe 1987A (solid line), 1999em (open triangle), 2004et (filled triangle), 2005cs (open circle), and 2008gz (open square).

(A color version of this figure is available in the online journal.)

considering the underlying assumption that all $\gamma$-rays emitted during the radioactive decay make the ejecta thermalized. For SN 2008in, the time interval spanned by the observations is about 416 days, where the first $\sim 100$ days are reserved for photospheric evolutionary processes. The average $V$ band magnitude during the nebular phase calculated using the data between +114 days and +416 days is $\sim 18.91$, which corresponds to the $V$ magnitude at $\sim+222$ days. Taking the extinction correction as $A_{V}=0.305 \pm 0.322$ mag (Section 5), a bolometric correction of $0.26 \pm 0.06 \mathrm{mag}$ (Hamuy 2001), and a distance modulus $30.6 \pm 0.2$, the derived tail luminosity at this fiducial time is $(3.02 \pm 1.95) \times 10^{40} \mathrm{erg} \mathrm{s}^{-1}$. Within the errors, this value is consistent with the bolometric flux at a comparable epoch, determined in Section 6. This implies that the amount of ${ }^{56} \mathrm{Ni}$ produced in this process is $M_{\mathrm{Ni}}=0.0157 \pm 0.0102 M_{\odot}$.

All Type IIP SNe show an inflection in the light curve during the transition from the plateau to the nebular phase. Statistically it has been shown that the steepness of the $V$-band light-curve slope (defined as $S=d m_{\mathrm{V}} / d t$ ) at the inflection time $\left(t_{i}\right)$ is anti-correlated with ${ }^{56} \mathrm{Ni}$ mass (Elmhamdi et al. 2003a). For SN 2008in, the $V$-band light curve with its well-sampled transition phase shows a value of steepness $S=0.151 \pm 0.044$ mag day $^{-1}$ (Figure 13 ) and the epoch of inflection is $t_{i} \approx+101.5$ days with respect to the date of discovery. This corresponds to
$M_{\mathrm{Ni}}=0.0175 \pm 0.002 M_{\odot}$. This result is consistent with the value measured using the Hamuy (2003) scheme. According to Elmhamdi et al. (2003a), the amount of ${ }^{56} \mathrm{Ni}$ can also be derived from the plateau absolute $V$-band magnitude using the relation $\log M_{\mathrm{Ni}}=-0.438 M_{V}\left(t_{i}-35\right)-8.46$. Here $M_{V}\left(t_{i}-35\right)$ is the absolute $V$ magnitude 35 days prior to the day of inflection. For SN 2008in, $M_{V}\left(t_{i}-35\right) \approx-15.23$, which again corresponds to a ${ }^{56} \mathrm{Ni}$ mass around $0.016 M_{\odot}$.

Comparison of the tail luminosity with that of SN $1987 \mathrm{~A}-\mathrm{a}$ well-studied proximate event-is also used for estimation of the ${ }^{56} \mathrm{Ni}$ mass. A linear least-squares fit on the nebular light-curve tail shows that at +222 days the luminosity of SN 2008in is about $1.54 \times 10^{40} \mathrm{erg} \mathrm{s}^{-1}$, while SN $1987 \mathrm{~A}$ had a luminosity $\sim 1.10 \times 10^{41} \mathrm{erg} \mathrm{s}^{-1}$ (Figure 12). Since the ${ }^{56} \mathrm{Ni}$ mass produced by SN $1987 \mathrm{~A}$ is about $0.075 M_{\odot}$, the amount of ${ }^{56} \mathrm{Ni}$ in the case of SN 2008in is $\left[(1.54 / 1.10) \times 10^{-1}\right] \times 0.075 \approx 0.0105 M_{\odot}$.

The above estimates are consistent with each other and hence we adopt a mean value for ${ }^{56} \mathrm{Ni}$ mass of $0.015 \pm 0.003 M_{\odot}$.

\subsection{Explosion Energy and Mass of Progenitor Star}

We use the radiation-hydrodynamical simulations of corecollapse IIP SNe by Dessart et al. (2010) to infer the explosion energy $\left(E_{0}\right.$ : kinetic plus thermal, expressed hereafter in units of $10^{51} \mathrm{erg}$, or foe). These simulations suggest that in a given 


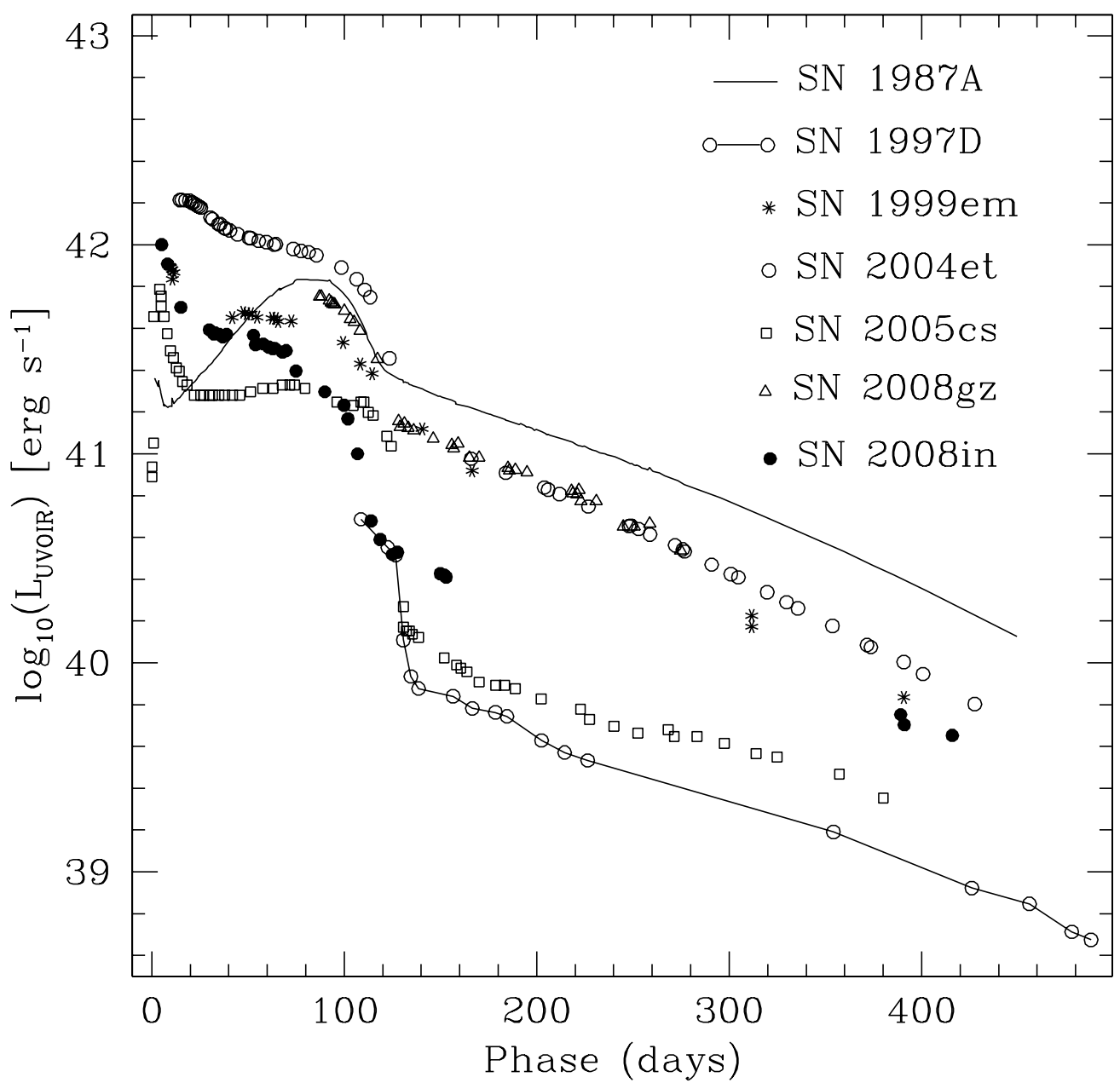

Figure 12. Comparison of the quasi-bolometric light curve of SN 2008in with the low-luminosity SNe 1997D, 2005cs; the normal SNe 1999em, 2004et, and 2008gz; and the peculiar Type II SN 1987A.

progenitor larger explosion energies yield larger ejecta velocities and this implies that the ejecta kinematics can be used to put a constraint on $E_{0}$. For SN 2008in, the expansion rate of the $\mathrm{H}$-rich progenitor envelope as derived from absorption minima in $\mathrm{H} \alpha$ at 15 days after shock breakout is about $6300 \mathrm{~km} \mathrm{~s}^{-1}$ (see Figure 8), which is higher than $4700 \mathrm{~km} \mathrm{~s}^{-1}$ (for SN 2005cs; Pastorello et al. 2009) and lower than $8800 \mathrm{~km} \mathrm{~s}^{-1}$ (for SN 1999em; Elmhamdi et al. 2003b). For the SNe 2005cs and $1999 \mathrm{em}$, the simulation results (for explosion of non-rotating solar metallicity pre-SN stars) predicted $E_{0} \sim 0.3$ foe and $\gtrsim 1$ foe respectively and these values are found to be consistent with that determined from the actual hydrodynamical modeling of the SN light curves, e.g., 0.4 foe (Utrobin \& Chugai 2008) and 0.3 foe (Pastorello et al. 2009) for SN 2005cs; 1.3 foe (Utrobin 2007) and 1.25 foe (Bersten et al. 2011) for SN 1999em. For SN 2008in, the simulations suggest $E_{0} \sim 0.5$ foe.

Accurate determinations of explosion parameters such as $E_{0}$, the ejected mass $\left(M_{\mathrm{ej}}\right)$, and the pre-SN radius $\left(R_{0}\right)$ of the progenitor require detailed hydrodynamical modeling of the light curves and spectra and this, being a non-trivial task (beyond the scope of this paper), has only been attempted for a few $\mathrm{SNe}$. In order to have an estimate of explosion parameters of SN 2008in here, we employ the analytical relations derived by Litvinova \& Nadezhin (1985) correlating the observed parameters $\left(M_{\mathrm{Vmp}}\right.$ : the mid-plateau absolute magnitude at $V ; v_{\text {mp }}$ : the mid-plateau photospheric velocity; and $\Delta t_{p}$ : the plateau duration) with the physical parameters $\left(E_{0}, M_{\mathrm{ej}}\right.$, and $R_{0}$ ) based on a grid of hydrodynamical models for different values of physical parameters for Type IIP SNe. We note, however, that these approximate formulae have limitations owing to the poorly measured observables and the simplified physical conditions such as non-inclusion of the effect of nickel heating, use of old opacity tables, neglecting the effect of line opacity, and using outdated pre-SN models (Smartt et al. 2009; Bersten et al. 2011 and references therein). As a result, only approximate values of the physical parameters can be derived using these relations. Fortunately, the observed parameters are derived very accurately for SN 2008in. The $\Delta t_{p}$ is $\sim 98$ days (Section 3.2), the $v_{\mathrm{mp}}$ is $2694 \pm 70^{27} \mathrm{~km} \mathrm{~s}^{-1}$, and the $M_{\mathrm{Vmp}}$ is estimated as $-15.32 \pm 0.38$ mag. ${ }^{28}$ Now, employing analytical relations, we estimate $E_{0} \sim 0.54$ foe, $M_{\mathrm{ej}} \sim 16.7 M_{\odot}$, and $R_{0} \sim 127 R_{\odot}$. The explosion energy derived in this way is consistent with that predicted from the hydrodynamical simulations of the ejecta kinematics.

\footnotetext{
27 This is the mean value of the velocities computed from the lines of Fe II $\lambda 4924, \lambda 5018$, and $\lambda 5169$ in the +54 day spectrum.

28 These observed values of $M_{\mathrm{Vmp}}$ and $v_{\mathrm{mp}}$ can be compared with the estimates derived empirically between the mass of ${ }^{56} \mathrm{Ni}$, and the $v_{\mathrm{mp}}$ and $M_{\mathrm{Vmp}}$ for a larger sample of IIP SNe (see Section 8.3 of Roy et al. 2011). Using the ${ }^{56} \mathrm{Ni}$ mass of $0.015 \pm 0.003 M_{\odot}$ we find $v_{\mathrm{mp}}=2916 \pm 220 \mathrm{~km} \mathrm{~s}^{-1}$ and $M_{\mathrm{Vmp}}=-15.37 \pm 0.23 \mathrm{mag}$, which are consistent with those measured observationally.
} 


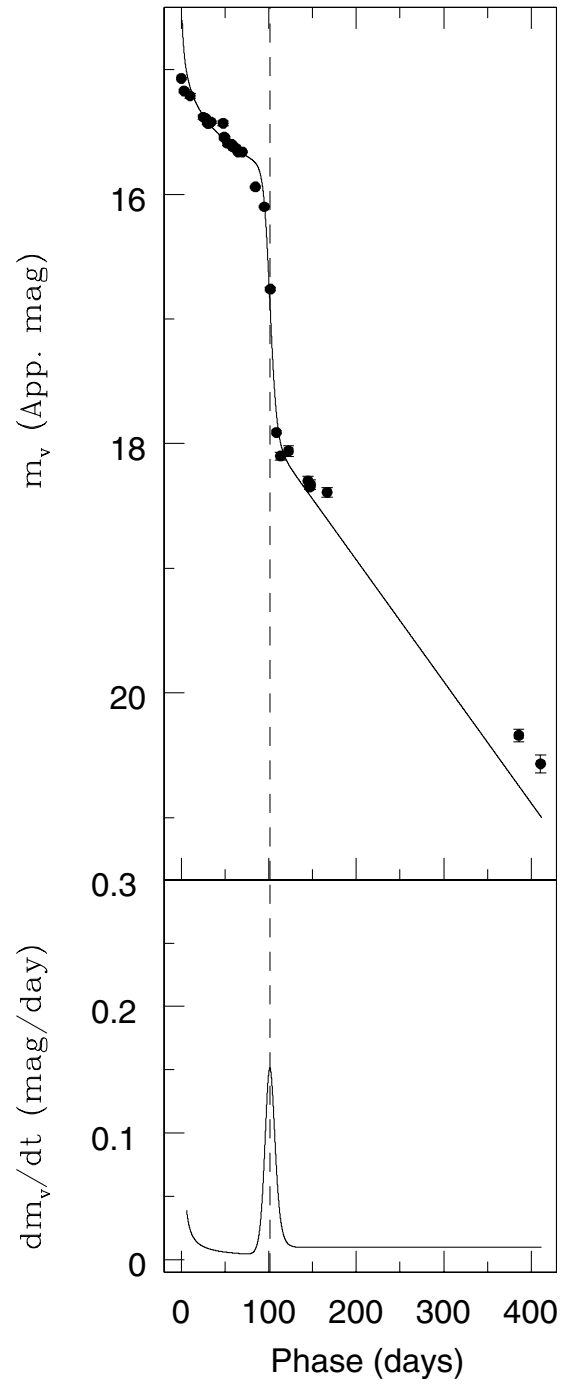

Figure 13. Determination of the steepness parameter from the apparent $V$-band light curve of SN 2008in. See the text for details.

The explosion energy of SN 2008in indicates that the event was less energetic than the standard IIP SNe 1999em, 2004et and more energetic than SN 2005cs. Assuming a net mass loss of $\sim 0.5 M_{\odot}$ due to stellar wind ${ }^{29}$ and accounting for a compact remnant with mass $\sim 1.5-2 M_{\odot}$ (Sahu et al. 2006), we find the initial mass of the progenitor to be $\leqslant 20 M_{\odot}$.

\section{DISCUSSION IN CONTEXT OF OTHER TYPE IIP SNe}

In Figure 14, we compare the light curve of SN 2008in in absolute $V$-band magnitude with a sample of 13 other wellstudied Type II SNe taken from the literature (see Misra et al. 2007 and Roy et al. 2011 for the references). The sample includes two low-luminosity Type IIP SNe 1997D, 2005cs; nine normal Type IIP SNe 1990E, 1992H, 1999gi, 1999em, 2003gd, 2004A, 2004dj, 2004et, 2008gz, and two peculiar Type II SNe 1987A, 1998A. It is seen that the Type II SNe show a wide range of mid-plateau luminosity, i.e., from -14 to $-17 \mathrm{mag}$. The data

\footnotetext{
29 SN 2008in was not detected in X-rays. The lack of X-ray emission can be used to constrain the mass-loss rate of the progenitor system that could be heated by the outgoing shock to X-ray emitting temperatures. Following the discussion in Immler et al. (2007) and references therein, an upper limit to the pre-SN mass-loss rate of $5 \times 10^{-6} M_{\odot} \mathrm{yr}^{-1}\left(v_{\mathrm{w}} / 10 \mathrm{~km} \mathrm{~s}^{-1}\right)$ with an uncertainty of a factor of two to three is obtained.
}

on low-luminosity SNe (see also Table 4 in Pastorello et al. 2006) indicate that their $M_{\mathrm{Vmp}}$ lie between -14 and $-15 \mathrm{mag}$, with the only exception of SN $2002 \mathrm{gd}$, whereas the other $\mathrm{SNe}$ are seen to lie between -16 and $-17 \mathrm{mag}$. With the $M_{\mathrm{Vmp}}$ of $-15.32 \mathrm{mag},{ }^{30}$ the SN 2008in presents another case which occupies the gap between low and normal luminosity Type IIP events.

The tail luminosity is an important indication of ejected nickel mass. The average tail luminosity of SN 2008in $\left(M_{\mathrm{v}} \sim-12.16\right)$ is nearly 2.3 mag brighter than those of the low-luminosity $\mathrm{SNe}$ 1997D and 2005cs ( - -9.6 mag), and about $1.3 \mathrm{mag}$ fainter than the normal Type IIP SNe $1992 \mathrm{H}, 2004 \mathrm{et}$, and $2008 \mathrm{gz}$ ( $\sim-13.5 \mathrm{mag})$. A close inspection shows that the average tail luminosity of SN 2008in is roughly similar to Type IIP SNe 2004dj in NGC 2403 and 2003gd in M74, whereas their plateau is brighter $(\sim 0.5-0.8 \mathrm{mag}$ at mid-plateau) than $\mathrm{SN} 2008 \mathrm{in}$. These comparisons of nebular phase luminosities with other $\mathrm{SNe}$ are consistent with a quite modest radioactive ${ }^{56} \mathrm{Ni}$ production for SN 2008in (Section 7.1).

The luminosity and shape of the tail depend on the ${ }^{56} \mathrm{Ni}$ mass and the radiant energy per unit ejected mass $\left(E / M_{\mathrm{ej}}\right)$. The first parameter determines the absolute magnitude, while the second determines its decay rate (see Turatto et al. 1998 and references therein). The measured values of ejected ${ }^{56} \mathrm{Ni}$ mass for SNe 2003gd, 2004dj, and 2008in are, respectively, $\sim 0.016$ (Hendry et al. 2005), 0.017 (Vinkó et al. 2006), and $0.015 M_{\odot}$. These are more than twice the ${ }^{56} \mathrm{Ni}$ amount synthesized by the low-luminosity SNe 1997D $\left(\sim 0.002 M_{\odot}\right.$; Turatto et al. 1998) and 2005cs (0.003-0.004 $M_{\odot}$; Pastorello et al. 2009), although at least three times less than the ${ }^{56} \mathrm{Ni}$ produced by normal Type IIP SNe such as $1992 \mathrm{H}\left(\sim 0.075 M_{\odot}\right.$; Clocchiatti et al. 1996), 2004et $\left(\sim 0.06 M_{\odot}\right.$; Sahu et al. 2006; Maguire et al. 2010), and 2008gz ( $\sim 0.05 M_{\odot}$; Roy et al. 2011). Similarly, the measured value of the $E / M_{\mathrm{ej}}$ ratio for SN 2008in is larger than that for the low-luminosity SN 1997D and lower than other Type IIP events. ${ }^{31}$

Though we have an approximate value of the pre-SN radius $\left(128 R_{\odot}\right)$, it can be seen that the progenitor radius of SN 2008in is smaller than or comparable to that of low-luminosity SNe 1997D $\left(\sim 300 R_{\odot}\right.$; Turatto et al. 1998), 2005cs (100-600 $R_{\odot}$; Pastorello et al. 2009; Utrobin \& Chugai 2008), 1999 br (114 $R_{\odot}$; Zampieri et al. 2003) and much smaller than that of normal Type IIP SNe like $1992 \mathrm{H}\left(>600 R_{\odot}\right.$; Clocchiatti et al. 1996), 2004et ( $\sim 530 R_{\odot}$; Misra et al. 2007). We also note that the SN 2008in progenitor is only slightly larger than the blue supergiant progenitors of SNe 1987A and 1998A (Pastorello et al. 2005).

Information about the metallicity of the SNe location is essential to constrain the triggering mechanism of the $\mathrm{SN}$ explosion (Heger et al. 2003). The oxygen abundance $[\mathrm{O} / \mathrm{H}]$ of SN 2008in is about 8.44 dex $^{32}$ which is marginally lower than

\footnotetext{
30 Considering a lower value of $A_{V}=0.139 \pm 0.002$, we get $M_{\mathrm{Vmp}}=-15.1 \pm 0.2$.

31 The measured value of the $E / M$ ratio for SN 2008in is $3.5 \times 10^{49} \mathrm{erg} M_{\odot}{ }^{-1}$, which is twice the $E / M$ ratio measured for SNe 1997D $\left(1.7 \times 10^{49} \operatorname{erg} M_{\odot}^{-1}\right.$; Turatto et al. 1998), but it is half those of SN $2008 \mathrm{gz}$ (7.3 $\times 10^{49} \mathrm{erg} M_{\odot}{ }^{-1}$; Roy et al. 2011), SN 2004dj $\left(7.3 \times 10^{49} \operatorname{erg} M_{\odot}{ }^{-1}\right.$; Vinkó et al. 2006), and SN 2004et (7.5 × $10^{49} \mathrm{erg}{M_{\odot}}^{-1}$; Sahu et al. 2006). Finally, it is barely a third the $E / M$ ratios of SNe 1987A $\left(11.3 \times 10^{49} \operatorname{erg} M_{\odot}^{-1}\right.$; Hamuy 2003) and 2003gd (13.0 $\times 10^{49} \operatorname{erg} M_{\odot}{ }^{-1}$; Hendry et al. 2005), and about seven times smaller than a luminous event like SN 1998A $\left(25.4 \times 10^{49} \operatorname{erg} M_{\odot}^{-1}\right.$; Pastorello et al. 2005).

32 Derived from the $[\mathrm{O} / \mathrm{H}]\left(=12+\log \left(N_{\mathrm{O}} / N_{\mathrm{H}}\right)\right)-M_{B}$ relation given by Pilyugin et al. (2004) for a given deprojected radius and galaxy type. The host of SN 2008in is a spiral galaxy of SBbc Type and the SN location is $\sim 1^{\prime} .8$
} 


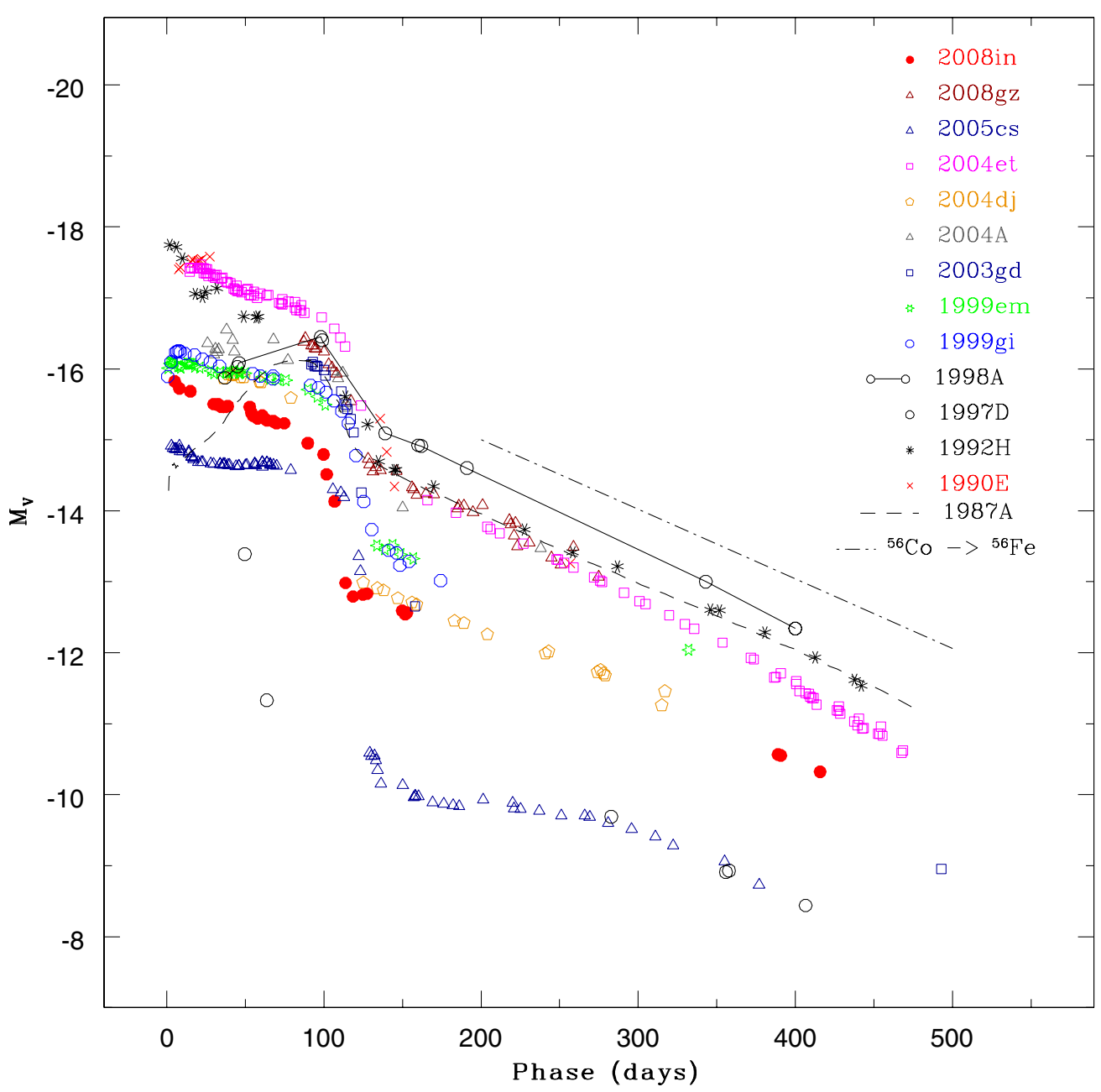

Figure 14. Comparison of the absolute $V$-band light curve of SN 2008in with the low-luminosity SNe 1997D, 2005cs; the normal SNe 1999em, 2004et, 2008gz, 2004dj, 2004A, 2003gd, 19992H, 1999E, and 1999gi; and the peculiar Type II SNe 1987A and 1998A. The decline rate of emission expected from radioactive decay of ${ }^{56} \mathrm{Ni}$ to ${ }^{56} \mathrm{Co}$ to ${ }^{56} \mathrm{Fe}$ is shown with the dot-dashed line.

(A color version of this figure is available in the online journal.)

the solar value of 8.65 dex (Asplund et al. 2009). A comparison ${ }^{33}$ of $[\mathrm{O} / \mathrm{H}]$ of all the events in our sample indicates that there is no clear trend in the type of event and the metallicity.

The photometric and spectroscopic comparisons of SN 2008in with some Type II SNe covering a wide range of physical parameters put observational constraints on the nature of the progenitor of this event, pointing toward a star that was more compact than a typical M-type red supergiant, and closer to a blue supergiant, maybe a yellow supergiant. However, the direct detection of the progenitors of a few faint SNe IIP in pre-explosion archive Hubble Space Telescope images seems to contradict this conclusion (see Smartt et al. 2009 for a review). Our spectroscopic study suggests that in SN 2008in the hydrogen envelope ejected by the explosion is smaller than in most Type II events but larger than that ejected in low-luminosity

away from the center of the host. This corresponds to a deprojected distance $\sim 7.03 \mathrm{kpc}$.

33 We have taken the $[\mathrm{O} / \mathrm{H}]$ value from Smartt et al. (2009), though for a few cases the values are estimated using the $[\mathrm{O} / \mathrm{H}]-M_{B}$ relation mentioned in Pilyugin et al. (2004). The [O/H] for normal Type IIP SNe 2008gz, 1999em and low-luminosity SNe $1999 \mathrm{eu}, 2005 \mathrm{cs}$ is about 8.6 . For the low-luminosity SNe 1994N, 1999br, 2001dc, 2003gd, 2004dj, 1997D, and the normal SN 2004et, it is about 8.4. In many cases, we have adopted the $[\mathrm{O} / \mathrm{H}]$ value from Smartt et al. (2009). For some cases we have estimated the value by adopting the $[\mathrm{O} / \mathrm{H}]-M_{B}$ relation mentioned in Pilyugin et al. (2004). events (such as SN 1997D, SN 1999br, and SN 1999eu). This may be due to a significant mass loss of the parent star in the latest stages of its life. This conclusion is also partially supported by the upper limit mass-loss rate of the progenitor revealed from the X-ray study (Section 7.2). In view of the upper mass limit of a $20 M_{\odot}$ progenitor, occurrence of the event in a sub-solar metallicity region, and following the evolution of a single massive star as a function of metallicity (Heger et al. 2003), we rule out the possibility of a "fall back of ejecta to BH" scenario in the case of SN 2008in, supporting the scenario of a weak explosion of a relatively compact progenitor.

\section{CONCLUSION}

Low-luminosity Type IIP SNe belong to a poorly known class of events due to the unknown nature of their progenitors as well as the explosion mechanism. Spectroscopic as well as photometric characteristics of these events are significantly different from the normal and peculiar Type IIP SNe. The number of such underluminous events discovered so far is relatively small. This could plausibly be a selection effect. If we confine the search of core-collapse $\mathrm{SNe}$ to a small volume, the majority of SNe discovered in the local universe are of Type IIP ( 48.2\%; Smith et al. 2010) and probably a large fraction of them would turn out to be underluminous. Incidentally 
SN 1999br, SN 2005cs, and SN 2009md are the only events of this group to have been discovered soon after the core-collapse and whose data are publicly available.

In this study, we have reported the results of an extensive photometric and spectroscopic follow-up campaign for SN 2008in in the ultraviolet, optical, and near-infrared domains. The SN was also observed (although not detected) at $\mathrm{X}$-ray and radio wavelengths. The $\mathrm{SN}$ was observed soon after its explosion, during the fast rise of the light curve to the optical maximum. Evidence for a shock breakout in SN 2008in was primarily derived through the analysis of the ROTSE-IIIb $R$-band light curve. An upper limit of 16.16 mag in the $R$ band was estimated about two days before the discovery.

Modeling the $R$-band light curve allowed us to estimate a reliable epoch of the shock breakout, with an uncertainty of about two days. The plateau phase in SN 2008in lasts about 98 days, which is marginally shorter than in normal Type IIP $\mathrm{SNe}$. The evolution of the bolometric light curve indicates that the event is somewhat in between the normal and faint Type IIP events. The luminosity of the nebular phase light curve indicates an ejected ${ }^{56} \mathrm{Ni}$ mass of $\sim 0.015 M_{\odot}$, a factor two higher than that derived for low-luminosity IIP SNe. The spectroscopic evolution of SN 2008in is similar to those of low-luminosity IIP SNe (1997D, 1999br, and 1999eu), indicated particularly by the strong presence of $\mathrm{Ba}$ II lines, the narrow line widths of $\mathrm{H} \alpha$ lines, and the expansion velocities of SN during the photospheric phases $\left(\sim 3000 \mathrm{~km} \mathrm{~s}^{-1}\right)$ and the nebular phases $\left(\sim 1200 \mathrm{~km} \mathrm{~s}^{-1}\right)$. The ejecta kinematics of SN 2008in are consistent with lessenergetic $\left(\sim 5 \times 10^{50} \mathrm{erg}\right)$ Type IIP SNe.

Spectroscopically SN 2008in appears to be like lowluminosity SNe IIP, but photometrically it appears close to a normal type IIP event.

From the light curve and spectra of SN 2008in, we could determine quite accurate values of the observed properties such as plateau duration, mid-plateau luminosity as well as the photospheric velocity, and this has helped us to comment on the properties of the explosion and the progenitor star. Using semi-analytical formulae by Litvinova \& Nadezhin (1985), we could estimate approximate values of the explosion energy $\sim 5.4 \times 10^{50} \mathrm{erg}$, the ejected mass $\sim 17 M_{\odot}$, and the pre-SN radius $\sim 127 R_{\odot}$. The explosion energy of SN 2008in is smaller than the normal $\left(\geqslant 10^{51} \mathrm{erg}\right)$ Type IIP events, although higher than that estimated in underenergetic $\left(\sim 10^{50} \mathrm{erg}\right) \mathrm{SNe}$ IIP. We could provide an upper limit to the mass-loss rate of the progenitor as $5 \times 10^{-6} M_{\odot} \mathrm{yr}^{-1}\left(v_{\mathrm{w}} / 10 \mathrm{~km} \mathrm{~s}^{-1}\right)$ whereas the upper limit for the main-sequence mass of the progenitor star is estimated as $20 M_{\odot}$.

We thank all the observers at the Aryabhatta Research Institute of Observational Sciences (ARIES) who provided their valuable time and support for the observations of this event. We are thankful to the observing staffs of ROTSE, REM, $2 \mathrm{~m}$ IGO, $3.6 \mathrm{~m}$ NTT, $6 \mathrm{~m}$ BTA, and $9.2 \mathrm{~m}$ HET for their kind cooperation in the observation of SN 2008in. This work was supported by the grant RNP 2.1.1.3483 of the Federal Agency of Education of Russia. Timur A. Fatkhullin and Alexander S. Moskvitin were supported by the grant of the President of the Russian Federation (MK-405.2010.2). This is also partially based on observations collected at the European Southern Observatory, Chile under the program 083.D-0970(A). Stefano Benetti and Filomena Bufano are partially supported by the PRIN-INAF 2009 with the project "Supernovae Variety and Nucleosynthesis Yields." The research of J. Craig Wheeler is supported in part by NSF Grant AST-0707669 and by the Texas Advanced Research Program grant ASTRO-ARP-0094. This research is supported by NASA grant NNX08AV63G and NSF grant PHY-0801007. This work is partially based on observations made with the REM Telescope, INAF Chile. This research has made use of data obtained through the High Energy Astrophysics Science Archive Research Center Online Service, provided by the NASA/Goddard Space Flight Center. We are indebted to the Indo-Russian (DST-RFBR) project No. RUSP836 (RFBR-08-02:91314) for the completion of this research work.

\section{REFERENCES}

Akerlof, C. W., et al. 2003, PASP, 115, 132

Alard, C. 2000, A\&AS, 144, 363

Arnett, W. D. 1980, ApJ, 237, 541

Arnett, W. D. 1996, Supernovae and Nucleosynthesis: An Investigation of the History of Matter, from the Big Bang to the Present (Princeton, NJ: Princeton Univ. Press)

Asplund, M., Grevesse, N., Sauval, A. J., \& Scott, P. 2009, ARA\&A, 47, 481

Barbon, R., Benetti, S., Rosino, L., Cappellaro, E., \& Turatto, M. 1990, A\&A, 237, 79

Benetti, S., et al. 2001, MNRAS, 322, 361

Bersten, M. C., Benvenuto, O., \& Hamuy, M. 2011, ApJ, 729, 61

Bessell, M. S., Castelli, F., \& Plez, B. 1998, A\&A, 333, 231

Brown, P. J. 2009, PhD thesis, Pennsylvania State Univ.

Brown, P. J., et al. 2009, AJ, 137, 4517

Burrows, D. N., et al. 2005, Space Sci. Rev., 120, 165

Chakraborti, S., Prabhu, T., Anupama, G. C., Kaur, A., Uday Kumar, G., \& Ray, A. 2008, CBET, 1638, 1

Chevalier, R. A. 1976, ApJ, 207, 872

Chugai, N. N., \& Utrobin, V. P. 2000, A\&A, 354, 557

Clocchiatti, A., et al. 1996, AJ, 111, 1286

Cowen, D. F., Franckowiak, A., \& Kowalski, M. 2010, Astropart. Phys., 33, 19

Dessart, L., Livne, E., \& Waldman, R. 2010, MNRAS, 408, 827

Dickey, J. M., \& Lockman, F. J. 1990, ARA\&A, 28, 215

Eldridge, J. J., Mattila, S., \& Smartt, S. J. 2007, MNRAS, 376, L52

Eldridge, J. J., \& Tout, C. A. 2004, MNRAS, 353, 87

Elmhamdi, A., Chugai, N. N., \& Danziger, I. J. 2003a, A\&A, 404, 1077

Elmhamdi, A., et al. 2003b, MNRAS, 338, 939

Falk, S. W., \& Arnett, W. D. 1977, ApJS, 33, 515

Foley, R. J. 2008, CBET, 1638, 2

Fransson, C., \& Chevalier, R. A. 1987, ApJ, 322, L15

Fransson, C., Lundqvist, P., \& Chevalier, R. A. 1996, ApJ, 461, 993

Fraser, M., et al. 2010, arXiv:1011.6558

Gezari, S., et al. 2008, ApJ, 683, L131

Grassberg, E. K., Imshennik, V. S., \& Nadyozhin, D. K. 1971, Ap\&SS, 10, 28

Hamuy, M. 2003, ApJ, 582, 905

Hamuy, M. 2005, in IAU Colloq. 192, Cosmic Explosions, On the 10th Anniversary of SN1993J, ed. J.-M. Marcaide \& K. W. Weiler (Berlin: Springer), 535

Hamuy, M., \& Pinto, P. A. 2002, ApJ, 566, L63

Hamuy, M., Suntzeff, N. B., Heathcote, S. R., Walker, A. R., Gigoux, P., \& Phillips, M. M. 1994, PASP, 106, 566

Hamuy, M. A. 2001, PhD thesis, Univ. Arizona

Heger, A., Fryer, C. L., Woosley, S. E., Langer, N., \& Hartmann, D. H. 2003, ApJ, 591, 288

Hendry, M. A., et al. 2005, MNRAS, 359, 906

Immler, S., et al. 2007, ApJ, 664, 435

Landolt, A. U. 2009, AJ, 137, 4186

Leonard, D. C., et al. 2002, PASP, 114, 35

Litvinova, I. Y., \& Nadezhin, D. K. 1985, Sov. Astron. Lett., 11, 145

Maguire, K., et al. 2010, MNRAS, 404, 981

Maund, J. R., \& Smartt, S. J. 2005, MNRAS, 360, 288

Maund, J. R., Smartt, S. J., \& Danziger, I. J. 2005, MNRAS, 364, L33

Misra, K., Pooley, D., Chandra, P., Bhattacharya, D., Ray, A. K., Sagar, R., \& Lewin, W. H. G. 2007, MNRAS, 381, 280

Nadyozhin, D. K. 2003, MNRAS, 346, 97

Nakano, S., Kadota, K., \& Wells, W. 2008, CBET, 1636, 1

Olivares, E. F., et al. 2010, ApJ, 715, 833

Pastorello, A., et al. 2004, MNRAS, 347, 74 
Pastorello, A., et al. 2005, MNRAS, 360, 950

Pastorello, A., et al. 2006, MNRAS, 370, 1752

Pastorello, A., et al. 2009, MNRAS, 394, 2266

Pilyugin, L. S., Vílchez, J. M., \& Contini, T. 2004, A\&A, 425, 849

Poole, T. S., et al. 2008, MNRAS, 383, 627

Press, W. H., Teukolsky, S. A., Vetterling, W. T., \& Flannery, B. P. (ed.) 1992, in Numerical Recipes in C. The Art of Scientific Computing (Cambridge: Cambridge Univ. Press)

Quimby, R. M., Wheeler, J. C., Höflich, P., Akerlof, C. W., Brown, P. J., \& Rykoff, E. S. 2007, ApJ, 666, 1093

Richmond, M. W., Treffers, R. R., Filippenko, A. V., Paik, Y., Leibundgut, B., Schulman, E., \& Cox, C. V. 1994, AJ, 107, 1022

Roming, P. W. A., et al. 2005, Space Sci. Rev., 120, 95

Roy, R., et al. 2011, MNRAS, 414, 167

Sahu, D. K., Anupama, G. C., Srividya, S., \& Muneer, S. 2006, MNRAS, 372 1315

Schawinski, K., et al. 2008, Science, 321, 223

Schlegel, D. J., Finkbeiner, D. P., \& Davis, M. 1998, ApJ, 500, 525

Smartt, S. J., Eldridge, J. J., Crockett, R. M., \& Maund, J. R. 2009, MNRAS, 395, 1409

Smith, N., Li, W., Filippenko, A. V., \& Chornock, R. 2011, MNRAS, 412, 1522

Stetson, P. B. 1987, PASP, 99, 191

Stockdale, C. J., et al. 2008, ATel, 1883, 1

Stockdale, C. J., et al. 2009, ATel, 1912, 1
Stritzinger, M. 2008, CBET, 1638, 3

Suntzeff, N. B., \& Bouchet, P. 1990, AJ, 99, 650

Terry, J. N., Paturel, G., \& Ekholm, T. 2002, A\&A, 393, 57

Tominaga, N., Blinnikov, S., Baklanov, P., Morokuma, T., Nomoto, K., \& Suzuki, T. 2009, ApJ, 705, L10

Turatto, M., Benetti, S., \& Cappellaro, E. 2003, in Proc. ESO/MPA/MPE, From Twilight to Highlight: The Physics of Supernovae, ed. W. Hillebrandt \& B. Leibundgut (Berlin: Springer), 200

Turatto, M., et al. 1998, ApJ, 498, L129

Utrobin, V. P. 2007, A\&A, 461, 233

Utrobin, V. P., \& Chugai, N. N. 2008, A\&A, 491, 507

Utrobin, V. P., Chugai, N. N., \& Botticella, M. T. 2010, ApJ, 723, L89

van Dokkum, P. G. 2001, PASP, 113, 1420

Van Dyk, S. D., et al. 2010, arXiv:1011.5873

Vinkó, J., et al. 2006, MNRAS, 369, 1780

Vollmann, K., \& Eversberg, T. 2006, Astron. Nachr., 327, 862

Waxman, E., Mészáros, P., \& Campana, S. 2007, ApJ, 667, 351

Weiler, K. W., Panagia, N., Montes, M. J., \& Sramek, R. A. 2002, ARA\&A, 40, 387

Yuan, F. 2010, PhD thesis, Univ. Michigan

Zampieri, L., Pastorello, A., Turatto, M., Cappellaro, E., Benetti, S., Altavilla, G., Mazzali, P., \& Hamuy, M. 2003, MNRAS, 338, 711

Zampieri, L., Shapiro, S. L., \& Colpi, M. 1998, ApJ, 502, L149

Zerbi, F. M., et al. 2004, Proc. SPIE, 5492, 1590 